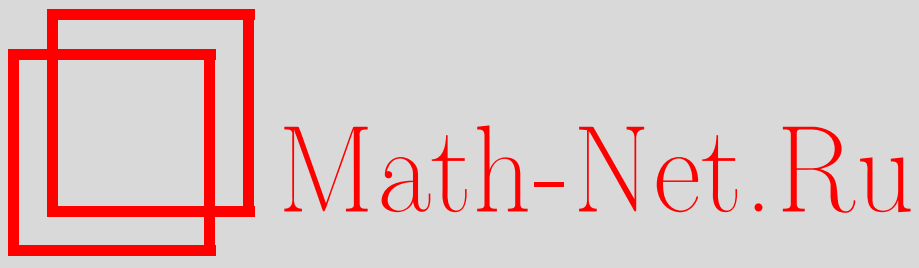

Г. И. Лаптев, Слабые решения квазилинейных параболических уравнений второго порядка с двойной нелинейностью, Матем. сб., 1997, том 188, номер 9, 83-112

DOI: https://doi.org/10.4213/sm258

Использование Общероссийского математического портала Math-Net.Ru подразумевает, что вы прочитали и согласны с пользовательским соглашением

http://www . mathnet.ru/rus/agreement

Параметры загрузки:

IP: 52.23 .180 .231

26 апреля 2023 г., $15: 22: 40$ 
УДК 517.9

\author{
Г.И. Лаптев
}

\title{
Слабые решения квазилинейных параболических уравнений второго порядка с двойной нелинейностью
}

В ограниченной области пространства $\mathbb{R}^{n}$ рассматривается первая краевая задача для уравнения

$$
\beta(u) \frac{\partial u}{\partial t}-\sum_{i=1}^{n} D_{i} A_{i}(t, x, u, D u)+A_{0}(t, x, u, D u)=0 .
$$

Предполагается, что функция $\beta(u)$ непрерьвна и удовлетворяет следующим условиям роста

$$
c|u|^{r-2} \leqslant \beta(u) \leqslant C\left(|u|^{r-2}+1\right), \quad r \geqslant 2 .
$$

Остальные коэффициенты удовлетворяют условиям, характерньм для теории монотонных операторов. Доказывается теорема существования глобального слабого решения.

Библиографйл: 18 названий.

В ограниченной области $(0, T) \times \Omega \subset \mathbb{R}^{n}, n \geqslant 1$, с кусочно-гладкой границей $S$ рассмотрим начально-краевую задачу

$$
\begin{gathered}
\beta(u) \frac{\partial u}{\partial t}-\sum_{i=1}^{n} D_{i} A_{i}(t, x, u, D u)+A_{0}(t, x, u, D u)=0 \\
u(0, x)=u_{0}(x) ;\left.\quad u\right|_{(0, T) \times S}=0 .
\end{gathered}
$$

Здесь переменная $t$ меняется от 0 до фиксированного числа $T>0 ; u=u(t, x)$ - искомая функция, определяемая на множестве $(t, x) \in \bar{Q}$, где $Q=(0, T) \times \Omega$; $D=\left(D_{1}, \ldots, D_{n}\right)$, где $D_{i}=\partial / \partial x_{i}$ для $i=1, \ldots, n ; u_{0}(x)$ - заданная функция. Условия на коэффициенты уравнения будут наложены позднее.

Начало теории разрешимости подобных задач было положено в работах [1], [2], где рассмотрен случай конкретных коэффициентов

$$
\beta(u)=|u|^{s} \quad(s>-1) ; \quad A_{i}=\left|D_{i} u\right|^{p-2} D_{i} u \quad(p>1) ; \quad A_{0}=0 .
$$

Эта теория подвергалась затем многочисленньм обобщениям. Их обзор, которьй будет дополнен далее, можно найти в работе [3]. Там же детально проанализирована связь с уравнениями, допускаюшими двойное вырождение, интерес к которым

Работа частично поддержана Российским фондом фундаментальных исследований (грант № 96-01-000-97).

(C) Г.И. ЛАПтев 1997 
значительно активизировался после обзорной статьи [4], где освешены разнообразные приложения таких уравнений. Во многих предыдущих статьях предполагается, что функции $\beta$ и $A_{i}$ связаны. Например, в основополагающей работе [5], где существенно расширен запас коэффициентов, требуется равенство

$$
A_{i}(t, x, u, \xi)=a_{i}(B(u), \xi), \quad \text { где } B(u)=\int_{0}^{u} \beta(s) d s .
$$

Аналогичные связи на рост функций $\beta$ и $A_{i}$ имеются также в статьях [6]-[8]. Попытки отказаться от них предприняты в работах [9]-[12]. При этом возникли ограничения другого рода. Так, в статьях [9], [10] при весьма слабых условиях на функцию $\beta(u)$ предполагается, что коэффициенты $\left\{A_{i}\right\}$ имеют вид градиента: $A_{i}(\xi)=\partial \varphi(\xi) / \partial \xi_{i}$, где $\varphi-$ выпуклая коэрцитивная функция. В [11] все коэффициенты подчинены выпуклым функциям. В [12], [13] предложены условия, обеспечивающие существование и единственность достаточно регулярного неотрицательного решения для неотрицательной начальной функции $u_{0}(x) \geqslant 0$. При этом уравнение представлено в форме так называемого уравнения с двойньм вырождением. Подводя итог, можно констатировать, что в ранее полученных результатах имеются ограничения, связанные с выбранным методом доказательства. В данной работе многие из этих ограничений снимаются или ослабляются и доказьвается сушествование слабого решения задачи (0.1) при условиях, характерных для теории монотонных операторов.

\section{§1. Формулировка результата}

Приведем предположения о функциях, входящих в уравнение (0.1).

$1 \beta$. Функция $\beta(u)$ определена на всей числовой оси, непрерывна и неотрицательна. С некоторым показателем $r \geqslant 2$ она удовлетворяет следующим условиям роста:

$$
c|u|^{r-2} \leqslant \beta(u) \leqslant C\left(|u|^{r-2}+1\right), \quad c>0 .
$$

Условия на коэффициенты $A_{i}$ формулируются длиннее. Предполагается, что функции $A_{i}(t, x, u, \xi)$ для $i=0,1, \ldots, n$ удовлетворяют условиям Каратеодори, т.е. они измеримы по аргументам $(t, x) \in Q$ для всех $u \in \mathbb{R}^{1}, \xi \in \mathbb{R}^{n}$ и непрерывны по $u, \xi$ для почти всех $(t, x)$. Кроме того, эти функции подчиняются следующим ограничениям.

1А. Условия роста. Для любого числа $\delta>0$ и некоторых положительных функций $C(\delta), C_{1}(\delta)$, определенных для $\delta>0$ :

$$
\left|A_{i}(t, x, u, \xi)\right| \leqslant c|\xi|^{p-1}+\delta\left(|u|^{q / p^{\prime}}+h(t)|u|^{r / p^{\prime}}\right)+C(\delta) h_{0}(t, x)
$$

где $p>1 ; q=p\left(1+\frac{r}{n}\right) ; h \in L^{p^{\prime}}(0, T), \frac{1}{p}+\frac{1}{p^{\prime}}=1 ; h_{0} \in L^{p^{\prime}}(Q) ; i=1, \ldots, n$;

$$
\left|A_{0}(t, x, u, \xi)\right| \leqslant \delta\left(|\xi|^{p / q^{\prime}}+|u|^{q-1}+h_{1}(t)|u|^{r / q^{\prime}}\right)+C_{1}(\delta) h_{2}(t, x),
$$

где $h_{1} \in L^{q^{\prime}}(0, T) ; h_{2} \in L^{q^{\prime}}(Q) ; \frac{1}{q}+\frac{1}{q^{\prime}}=1$. 
2А. Условие параболичности. Для почти всех $(t, x) \in Q$ и всех $u \in \mathbb{R}^{1}, \xi, \eta \in \mathbb{R}^{n}$

$$
\sum_{i=1}^{n}\left[A_{i}(t, x, u, \xi)-A_{i}(t, x, u, \eta)\right]\left(\xi_{i}-\eta_{i}\right)>0 \quad(\xi \neq \eta)
$$

3А. Условие коэрцитивности. Для любого числа $\delta>0$ и некоторой положительной функции $C_{2}(\delta)$, определенной для $\delta>0$ :

$\sum_{i=1}^{n} A_{i}(t, x, u, \xi) \xi_{i}+A_{0}(t, x, u, \xi) u \geqslant c_{0}|\xi|^{p}-\delta g(x)|u|^{p}-\delta g_{1}(t)|u|^{r}-C_{2}(\delta) g_{2}(t, x)$,

где $c_{0}>0 ; g_{1} \in L^{1}(0, T) ; g_{2} \in L^{1}(Q) ; g(x) \in L^{s}(\Omega)$, где $s=n / p$ для $p<n, s>1$ для $p=n, s=1$ для $p>n$.

Сделаем следующее замечание. Часто в уравнении (0.1) выделяют правую часть в форме заданной функции, т.е. пишут уравнение в виде $L u=f$. Условия $1 \mathrm{~A}-3 \mathrm{~A}$ представлены в такой форме, что заданную функцию $f$ можно включить в коэффициенты уравнения, оценив ее слагаемьми $h_{0}, h_{2}$ и $g_{2}$.

Цель работы - установить сушествование слабого решения задачи (0.1). При формулировке результата используются традиционные обозначения пространств абстрактных функций, заданных на отрезке $[0, T]$ и принимающих значения в некотором банаховом пространстве. Свойства таких пространств изложены в монографиях [1], [14]. Символом $(u, v)=\int_{\Omega} u v d x$ обозначается двойственность между пространствами $L^{p^{\prime}}(\Omega)$ и $L^{p}(\Omega)$, а символом $\langle u, v\rangle=\int_{Q} u v d Q-$ двойственность между пространствами $L^{p^{\prime}}(Q)$ и $L^{p}(Q)$. В последуюшем изложении основную роль играют банаховы пространства $X_{0}=L^{\infty}\left(0, T ; L^{r}(\Omega)\right)$ и $X_{1}=L^{p}(0, T ; \stackrel{\circ}{W} \underset{p}{1}(\Omega))$, наделенные нормами:

$$
\|u\|_{X_{0}}=\underset{t}{\operatorname{ess} \sup }\|u(t, x)\|_{L^{r}(\Omega)} ; \quad\|u\|_{X_{1}}=\|D u(t, x)\|_{L^{p}(Q)},
$$

а также их пересечение $X=X_{0} \cap X_{1}$, наделенное нормой $\|u\|_{X}=\|u\|_{X_{0}}+\|u\|_{X_{1}}$. Часто используются также пространство $X_{1}^{*}=L^{p^{\prime}}\left(0, T ; W_{p^{\prime}}^{-1}(\Omega)\right)$, сопряженное к $X_{1}$, и пространство $X^{*}$, сопряженное к $X$.

Введем функцию $B(u)=\int_{0}^{u} \beta(s) d s$ и перепишем уравнение $(0.1)$ в такой форме

$$
\begin{gathered}
\frac{\partial}{\partial t} B(u)-\sum_{i=1}^{n} D_{i} A_{i}(t, x, u, D u)+A_{0}(t, x, u, D u)=0 ; \\
B(u(0, x))=B\left(u_{0}(x)\right) ;\left.\quad u\right|_{S}=0 .
\end{gathered}
$$

Главным результатом работы является следуюшее утверждение.

Теорема 1.1. Пусть выполнены условия $1 \beta$ и $1 \mathrm{~A}-3 \mathrm{~A}$. Если $u_{0}(x) \in L^{r}(\Omega)$, то задача (1.1) имеет решение и $(t, x)$ со следующими свойствами:

(a) $u \in X \subset L^{q}(Q) ; A_{i}(t, x, u, D u) \in L^{p^{\prime}}(Q) \quad(i=1, \ldots, n) ; A_{0}(t, x, u, D u) \in$ $L^{q^{\prime}}(Q)$ 
(b) производная $\frac{\partial}{\partial t} B(u)$ определена как әлемент пространства $\mathscr{D}^{\prime}(0, T$; $\left.L^{r^{\prime}}(\Omega)\right)$ и при этом $\frac{\partial}{\partial t} B(u) \in X^{*} ;$

(c) функиия $u(t, x)$ для любого элемента $w \in X \subset L^{q}(Q)$ удовлетворяет уравнению

$$
\left\langle\frac{\partial}{\partial t} B(u), w\right\rangle+\sum_{i=1}^{n}\left\langle A_{i}(t, x, u, D u), D_{i} w\right\rangle+\left\langle A_{0}(t, x, u, D u), w\right\rangle=0 ;
$$

(d) $B(u(t, x)) \in C\left([0, T], w-L^{r^{\prime}}(\Omega)\right)$, әде символ $w$ - означает слабую топологию пространства $L^{r^{\prime}}(\Omega)$, и при этом как непрерывная функция принимает значение $B(u(0, x))=B\left(u_{0}(x)\right)$. $B$ этом смысле понимается начальное условие задачи.

\section{§2. Вспомогательные утверждения}

Далее через $c, C$ обозначаются, вообше говоря, различные положительные постоянные, которые зависят только от исходных данных рассматриваемой задачи. Отметим некоторые свойства функции $B(u)=\int_{0}^{u} \beta(s) d s$.

Лемма 2.1. Функиия $B(u) \in C^{1}\left(\mathbb{R}^{1}\right)$, строго монотонно возрастает и осуществляет гомеоморфизм вещественной оси $\mathbb{R}^{1}$ на себя. Она удовлетворяет также следующим условиям роста:

(a) $c|u|^{r-1} \leqslant|B(u)| \leqslant C\left(|u|^{r-1}+1\right)$;

(b) $c|u-v|^{r} \leqslant(u-v)(B(u)-B(v))$;

(c) $|B(u)-B(v)| \leqslant C|u-v|\left(1+|u|^{r-2}+|v|^{r-2}\right)$.

ДоказАТЕльство. Так как $B^{\prime}(u)=\beta(u)>0$ для $u \neq 0$, то функция $B(u)$ строго монотонно возрастает. Неравенства (а) являются непосредственньм следствием условий роста $1 \beta$ на функцию $\beta(u)$. Из левой части этих неравенств вытекает, что образ функции $B(u)$ заполняет всю ось $\mathbb{R}^{1}$. Так как $B(u)$ непрерьвна, то она отображает ось $\mathbb{R}^{1}$ на себя гомеоморфно. Для проверки соотношения (b) используем формулу Адамара

$$
B(u)-B(v)=(u-v) \int_{0}^{1} \beta(v+\tau(u-v)) d \tau .
$$

Отсюда по условию $1 \beta$

$$
(u-v)(B(u)-B(v)) \geqslant(u-v)^{2} \int_{0}^{1} c|v+\tau(u-v)|^{r-2} d \tau .
$$

Используя известное неравенство

$$
\int_{0}^{1}|v+\tau(u-v)|^{r-2} d \tau \geqslant c|u-v|^{r-2} \quad(c>0)
$$

получим утверждение (b) леммы. Аналогично, из формулы Адамара и условия $1 \beta$ получается неравенство (c), что завершает доказательство леммы.

В дальнейшем значительную роль играет функция $\Gamma(u)=\int_{0}^{u} s \beta(s) d s$. Ее свойства собраны в следующих далее утверждениях. 
ЛЕмма 2.2. Функиия $\Gamma(u) \in C^{1}\left(\mathbb{R}^{1}\right)$ и удовлетворяет следующим условиям pocma:

$$
c|u|^{r} \leqslant \Gamma(u) \leqslant C\left(|u|^{r}+1\right)
$$

Доказательство следует непосредственно из определения функции $Г(u)$ и условия $1 \beta$.

При использовании метода монотонности в параболических уравнениях важную роль играет вьпуклость некоторых функций, так как они порождают выпуклые функционалы. Введенные выше функции $B(u), \Gamma(u)$ выпуклыми быть не обязаны. Однако некоторая их композиция дает выпуклую функцию. Построим такую композицию. По лемме 2.1 функция $B(u)$ осуществляет гомеоморфизм вещественной оси $\mathbb{R}^{1}$ на себя. В частности, она имеет обратную функцию, которую обозначим $\alpha(U)$, так что уравнение $U=B(u)$ разрешимо для любого $U \in \mathbb{R}^{1}$ в форме $u=\alpha(U)$. Составим композицию $\gamma(U) \equiv \Gamma(\alpha(U))$, так что $\gamma(B(u))=\Gamma(u)$.

ЛЕМма 2.3. Функиия $\gamma(U) \in C^{1}\left(\mathbb{R}^{1}\right)$, является вьлпуклой и удовлетворяет следующему условию роста: $\gamma(U) \leqslant C\left(|U|^{r^{\prime}}+1\right)$, әде $\frac{1}{r}+\frac{1}{r^{\prime}}=1$.

ДокАЗАТЕЛЬСТво. Согласно общей теории обратных функций

$$
\gamma^{\prime}(U)=\frac{d \Gamma}{d \alpha} \cdot \frac{d \alpha}{d U}=\frac{d \Gamma}{d u}\left(\frac{d B}{d u}\right)^{-1}=u \beta(u)[\beta(u)]^{-1}=u=\alpha(U)
$$

Функция $\alpha U$, являясь обратной к строго монотонно возрастающей функции $B(u)$, сама монотонно возрастает, что является достаточным для выпуклости функции $\gamma(U)$. Далее, первое из неравенств леммы 2.1 можно представить в таком виде: $c|\alpha(U)|^{r-1} \leqslant|U|$. Аналогично, последнее неравенство леммы 2.2 запишем так: $\Gamma(\alpha(U)) \leqslant C\left(|\alpha(U)|^{r}+1\right)$. Отсюда

$$
\gamma(U)=\Gamma(\alpha(U)) \leqslant C\left[\left(|\alpha(U)|^{r-1}\right)^{r^{\prime}}+1\right] \leqslant C\left(|U|^{r^{\prime}}+1\right) .
$$

Это завершает доказательство леммы.

По коэффициентам рассматриваемого уравнения введем функцию

$$
H(t, x, u, \eta, \xi)=\sum_{i=1}^{n}\left[A_{i}(t, x, u, \xi)-A_{i}(t, x, u, \eta)\right]\left(\xi_{i}-\eta_{j}\right)
$$

считая ее определенной для $(t, x) \in Q, u \in \mathbb{R}^{1}, \xi, \eta \in \mathbb{R}^{n}$.

Лемма 2.4. На каждом ограниченном множестве вида $|u| \leqslant M,|\eta| \leqslant M_{1}$ справедливо неравенство

$$
H(t, x, u, \eta, \xi) \geqslant c(t, x)|\xi-\eta| \quad\left(|\xi| \geqslant 2+M_{1}\right),
$$

где функиия $c(t, x)$ положсительна для почти всех $(t, x) \in Q$. Она может зависеть от выбранных постоянных $M, M_{1}$, но не зависит от аргумента $\xi \in \mathbb{R}^{n}$. 
ДокАЗАТЕЛЬСТво. Используя представление $\xi=\eta+\rho \zeta^{0}$, где $\left|\zeta^{0}\right|=1$, $\rho=|\xi-\eta|$, введем функцию $h(\rho)=H\left(t, x, u, \eta, \eta+\rho \zeta^{0}\right)$, считая все аргументы фиксированными, кроме $\rho>0$. Согласно определению

$$
h(\rho)=\sum_{i=1}^{n}\left[A_{i}\left(t, x, u, \eta+\rho \zeta^{0}\right)-A_{i}(t, x, u, \eta)\right] \rho \zeta_{i}^{0},
$$

что можно представить в такой форме:

$$
\begin{aligned}
h(\rho)= & \sum_{i=1}^{n}\left[A_{i}\left(t, x, u, \eta+\rho \zeta^{0}\right)-A_{i}\left(t, x, u, \eta+\zeta^{0}\right)\right] \rho \zeta_{i}^{0} \\
& +\sum_{i=1}^{n}\left[A_{i}\left(t, x, u, \eta+\zeta^{0}\right)-A_{i}(t, x, u, \eta)\right] \rho \zeta_{i}^{0} \equiv \sigma_{1}+\sigma_{2} .
\end{aligned}
$$

Пусть $\xi^{0}=\eta+\zeta^{0}$, тогда $\xi-\xi^{0}=(\rho-1) \zeta^{0}$, так что $\rho \zeta^{0}=\frac{\rho}{\rho-1}\left(\xi-\xi^{0}\right)$. Следовательно,

$$
\sigma_{1}=\sum_{i=1}^{n}\left[A_{i}(t, x, u, \xi)-A_{i}\left(t, x, u, \xi^{0}\right)\right]\left(\xi_{i}-\xi_{i}^{0}\right) \frac{\rho}{\rho-1}>0
$$

если $\rho \geqslant 2$, в силу условия параболичности $2 \mathrm{~A}$. В результате получается оценка $h(\rho)=\sigma_{1}+\sigma_{2} \geqslant \sigma_{2}$. Заметим, что $\sigma_{2}=\rho h(1)$, так что $h(\rho) \geqslant \rho h(1)$ при $\rho \geqslant 2$. Это неравенство в терминах функции $H$ принимает такой вид

$$
H(t, x, u, \eta, \xi) \geqslant|\xi-\eta| H\left(t, x, u, \eta, \eta+\zeta^{0}\right),
$$

где $\xi=\eta+\rho \zeta^{0},\left|\zeta^{0}\right|=1, \rho \geqslant 2$. Введем константу

$$
\begin{gathered}
c(t, x)=\min _{R} H\left(t, x, u, \eta, \eta+\zeta^{0}\right), \\
R=\left\{|u| \leqslant M,|\eta| \leqslant M_{1},\left|\zeta^{0}\right|=1\right\},
\end{gathered}
$$

считая постоянные $M, M_{1}$ фиксированными. В силу условия Каратеодори функция $H$ непрерывна по аргументам $u, \eta, \xi=\eta+\zeta^{0}$ для почти всех $(t, x) \in Q$. Выберем именно такую точку $(t, x)$. Так как множество $R$ ограниченно и замкнуто, то введенный минимум $c(t, x)$ достигается, т.е. найдутся аргументы $u_{m}, \eta_{m}, \zeta_{m}^{0}$, для которых $c(t, x)=H\left(t, x, u_{m}, \eta_{m}, \eta_{m}+\zeta_{m}^{0}\right)$. Так как последние аргументы $\eta_{m}$ и $\eta_{m}+\zeta_{m}^{0}$ различны, то по условию параболичности 2 А выполняется строгое неравенство $c(t, x)>0$. Итак, функция $c(t, x)$ положительна для почти всех $(t, x) \in Q$. Подставляя ее в неравенство (2.1), получим $H \geqslant c(t, x)|\xi-\eta|$. Это и есть утверждение леммы.

Лемма 2.5. Пространство $X$ непрерывно вложено в пространство $L^{r}(Q) \cap L^{q}(Q)$, где $q=p\left(1+\frac{r}{n}\right)$. 
ДокАЗАТЕЛЬство. Если $q \leqslant r$, то утверждение очевидно, так как пространство $X$ является частью пространства $L^{\infty}\left(0, T ; L^{r}(\Omega)\right)$, которое непрерывно вложено в $L^{r}(Q)$. Поэтому рассмотрим случай $q>r$. Воспользуемся интерполяционным неравенством $[15$, с. 79$]$ :

$$
\|u\|_{L^{q}(\Omega)} \leqslant C\|D u\|_{L^{p}(\Omega)}^{\alpha}\|u\|_{L^{r}(\Omega)}^{1-\alpha}, \quad \alpha=\left(\frac{1}{r}-\frac{1}{q}\right)\left(\frac{1}{n}-\frac{1}{p}+\frac{1}{r}\right)^{-1} .
$$

С учетом выбора $q=p\left(1+\frac{r}{n}\right)$ получаем $\alpha=\frac{n}{r+n}$, так что $\alpha q=p ;(1-\alpha) q=$ $q-p$. Возводя в степень $q$ интерполяционное неравенство и интегрируя его по отрезку $[0, T]$, считая $u=u(t, x)$, получим

$$
\int_{0}^{T}\|u\|_{L^{q}(\Omega)}^{q} d t=\|u\|_{L^{q}(Q)}^{q} \leqslant C \int_{0}^{T}\|D u\|_{L^{p}(\Omega)}^{p} \cdot\|u\|_{L^{r}(\Omega)}^{q-p} d t
$$

Предполагая, что $u \in X$, получим

$$
\|u\|_{L^{q}(Q)}^{q} \leqslant C \underset{t}{\operatorname{ess} \sup }\|u\|_{L^{r}(\Omega)}^{q-p} \cdot\|D u\|_{L^{p}(Q)}^{p} \leqslant C\|u\|_{X}^{q} .
$$

Отсюда следует утверждение леммы для рассматриваемого случая $q>r$, что завершает доказательство.

ЛЕмма 2.6. Пусть $E$ - произвольное измеримое подмножество области $Q$ и пусть выполнены условия роста $1 \mathrm{~A}$. Тогда справедливы следующие оценки, в которых постоянная $C$ не зависит ни от множества $E$, ни от выбранных функций $u, v \in X$.

a) Ecли $i=1,2, \ldots, n$, mо $A_{i}(t, x, u, D v) \in L^{p^{\prime}}(Q)$ и при этом

$$
\begin{aligned}
& \left(\int_{E}\left|A_{i}(t, x, u, D v)\right|^{p^{\prime}} d Q\right)^{1 / p^{\prime}} \\
& \quad \leqslant C\left(\|D v\|_{L^{p}(E)}^{p-1}+\delta\|u\|_{X}^{q / p^{\prime}}+\delta\|u\|_{X}^{r / p^{\prime}}+C(\delta)\left\|h_{0}\right\|_{L^{p^{\prime}(E)}}\right) .
\end{aligned}
$$

b) Eсли $i=0$, mо $A_{0}(t, x, u, D u) \in L^{q^{\prime}}(Q)$ и при этом

$$
\begin{aligned}
& \left(\int_{E}\left|A_{0}(t, x, u, D u)\right|^{q^{\prime}} d Q\right)^{1 / q^{\prime}} \\
& \quad \leqslant C\left[\delta\left(\|u\|_{X}^{p / q^{\prime}}+\|u\|_{X}^{q-1}+\|u\|_{X}^{r / q^{\prime}}\right)+C_{1}(\delta)\left\|h_{2}\right\|_{L^{q^{\prime}}(E)}\right]
\end{aligned}
$$


ДокАЗАТЕЛЬСтво. Применим элементарное неравенство, справедливое для любых чисел $a, b \geqslant 0$ :

$$
(a+b)^{m} \leqslant C\left(a^{m}+b^{m}\right), \quad m>0 .
$$

Используя условия роста $1 \mathrm{~A}$, получаем для $i=1,2, \ldots, n$

$$
\left|A_{i}(t, x, u, D v)\right| \leqslant c|D v|^{p-1}+\delta|u|^{q / p^{\prime}}+\delta h(t)|u|^{r / p^{\prime}}+C(\delta) h_{0}(t, x) .
$$

Возводя в степень $p^{\prime}$ и применяя неравенство (2.2), получим

$$
\left|A_{i}(t, x, u, D v)\right|^{p^{\prime}} \leqslant C\left(c^{p^{\prime}}|D v|^{p}+\delta^{p^{\prime}}|u|^{q}+\delta^{p^{\prime}}|h|^{p^{\prime}} \cdot|u|^{r}+C^{p^{\prime}}(\delta)\left|h_{0}\right|^{p^{\prime}}\right) .
$$

Интегрируя это неравенство по множеству $E \subset Q$, найдем

$$
\begin{aligned}
\int_{E}\left|A_{i}(t, x, u, D v)\right|^{p^{\prime}} d Q & \\
\leqslant C\left(c^{p^{\prime}} \int_{E}|D v|^{p} d Q+\delta^{p^{\prime}} \int_{E}|u|^{q} d Q\right. & +\delta^{p^{\prime}} \int_{E}|h|^{p^{\prime}}|u|^{r} d Q \\
& \left.+C^{p^{\prime}}(\delta) \int_{E}\left|h_{0}\right|^{p^{\prime}} d Q\right) .
\end{aligned}
$$

Оценим первое слагаемое с множителем $\delta$ :

$$
\int_{E}|u|^{q} d Q \leqslant \int_{Q}|u|^{q} d Q=\|u\|_{L^{q}(Q)}^{q} \leqslant C\|u\|_{X}^{q}
$$

Здесь использована лемма 2.5 о непрерывности вложения $X \subset L^{q}(Q)$. Оценим второе слагаемое с множителем $\delta$ :

$$
\begin{aligned}
\int_{E}|h(t)|^{p^{\prime}}|u|^{r} d Q & \leqslant \int_{0}^{T} \int_{\Omega}|h(t)|^{p^{\prime}}|u|^{r} d x d t \leqslant \underset{t}{\operatorname{ess} \sup } \int_{\Omega}|u|^{r} d x \cdot \int_{0}^{T}|h(t)|^{p^{\prime}} d t \\
& =\|u\|_{L^{\infty}\left(0, T ; L^{r}(\Omega)\right)}^{r}\|h\|_{L^{p^{\prime}(0, T)}}^{p^{\prime}} \leqslant\|h\|_{L^{p^{\prime}(0, T)}}^{p^{\prime}} \cdot\|u\|_{X}^{r} .
\end{aligned}
$$

Полученные оценки подставим в неравенство (2.3):

$$
\begin{aligned}
& \int_{E}\left|A_{i}(t, x, u, D v)\right|^{p^{\prime}} d Q \\
& \quad \leqslant C\left(c^{p^{\prime}}\|D v\|_{L^{p}(E)}^{p}+C \delta^{p^{\prime}}\|u\|_{X}^{q}+\delta^{p^{\prime}}\|h\|_{L^{p^{\prime}(0, T)}}^{p^{\prime}} \cdot\|u\|_{X}^{r}+C^{p^{\prime}}(\delta)\left\|h_{0}\right\|_{L^{p^{\prime}(E)}}^{p^{\prime}}\right) .
\end{aligned}
$$

Возводя в степень $1 / p^{\prime}$ и снова применяя неравенство (2.2), получим оценку леммы для индексов $i=1, \ldots, n$. Полагая в ней $E=Q$, убедимся, что $A_{i}(t, x, u, D v) \in$ $L^{p^{\prime}}(Q)$, что доказывает пункт а) леммы.

Используя условия роста $1 \mathrm{~A}$ для $i=0$, запишем оценку

$$
\left|A_{0}(t, x, u, D u)\right| \leqslant \delta\left(|D u|^{p / q^{\prime}}+|u|^{q-1}+h_{1}(t)|u|^{r / q^{\prime}}\right)+C_{1}(\delta) h_{2}(t, x),
$$


что после возведения в степень $q^{\prime}$ и интегрирования по множеству $E$ дает

$$
\begin{aligned}
\int_{E}\left|A_{0}(t, x, u, D u)\right|^{q^{\prime}} d Q & \\
\leqslant C\left[\delta ^ { q ^ { \prime } } \left(\int_{E}|D u|^{p} d Q+\int_{E}|u|^{q} d Q+\right.\right. & \left.\int_{E}\left|h_{1}(t)\right|^{q^{\prime}}|u|^{r} d Q\right) \\
& \left.+C_{1}^{q^{\prime}}(\delta) \int_{E}\left|h_{2}\right|^{q^{\prime}} d Q\right] .
\end{aligned}
$$

Первое слагаемое с множителем $\delta$ оценивается просто:

$$
\int_{E}|D u|^{p} d Q \leqslant \int_{Q}|D u|^{p} d Q=\|u\|_{X_{1}}^{p} \leqslant\|u\|_{X}^{p}
$$

Оставшиеся два слагаемых с множителем $\delta$ уже оценивались ранее, так что можем записать

$$
\int_{E}|u|^{q} d Q \leqslant C\|u\|_{X}^{q} ; \quad \int_{E}\left|h_{1}(t)\right|^{q^{\prime}}|u|^{r} d Q \leqslant\left\|h_{1}\right\|_{L^{p^{\prime}}(0, T)}^{q^{\prime}} \cdot\|u\|_{X}^{r} .
$$

Подставляя в неравенство (2.4) и возводя результат в степень $1 / q^{\prime}$, получим последнее утверждение леммы, чем завершается ее доказательство.

Пусть задана последовательность функций $u_{N} \in X(N=1,2, \ldots)$. Введем обозначения

$$
w_{i N}=A_{i}\left(t, x, u_{N}, D u_{N}\right) \quad(i=0,1, \ldots, n)
$$

Лемма 2.7. Пусть $\left\|u_{N}\right\|_{X} \leqslant C$. Тогда последовательности $w_{i N}$ равномерно ограничены в норме пространства $L^{p^{\prime}}(Q)$ для $i=1,2, \ldots, n$ и в норме пространства $L^{q^{\prime}}(Q)$ для $i=0$. Более того, из последовательности $u_{N}$ можнно выделить подпоследовательность, снова обозначаемую $u_{N}$, такую, что для некоторых әлементов $w_{i} \in L^{p^{\prime}}(Q) \quad(i=1, \ldots, n)$ u $w_{0} \in L^{q^{\prime}}(Q)$ справедливы соотношения: $w_{i N} \rightarrow w_{i}$ слабо в пространстве $L^{p^{\prime}}(Q)$ для $i=1,2, \ldots, n u w_{0 N} \rightarrow w_{0}$ слабо в пространстве $L^{q^{\prime}}(Q)$ при $N \rightarrow \infty$.

ДокАЗАТЕльство. Ограниченность последовательностей $w_{i N}$ в указанных пространствах вытекает из леммы 2.6, в которой следует положить $E=Q$, $u=v=u_{N}$. Сформулированные в лемме сходимости являются следствием реффлексивности пространств $L^{p^{\prime}}(Q)$ и $L^{q^{\prime}}(Q)$.

Лемма 2.8. Пусть $\left\|u_{N}\right\|_{X} \leqslant C$ и $u_{N} \rightarrow$ и почти всюду в $Q$ при $N \rightarrow \infty$. Тогда для каждого $i=1,2, \ldots, n$ и фиксированного $v \in X$ последовательность $z_{i N}=A_{i}\left(t, x, u_{N}, D v\right)$ сходится $\kappa$ функиии $z_{i}=A_{i}(t, x, u, D v)$ в норме пространства $L^{p^{\prime}}(Q)$. При этом оператор $a_{i}(u, v)=A_{i}(t, x, u, D v)$ непрерввен по аргументу $v$ как оператор из $X$ в $L^{p^{\prime}}(Q)$ для каждого фиксированного $u \in X$. 
ДокАЗАТЕЛЬСТво. Так как $u_{N} \rightarrow u$ почти всюду в $Q$, то $z_{i N} \rightarrow z_{i}$ почти всюду в $Q$. Убедимся, что последовательность $z_{i N}$ сходится к функции $z_{i}$ в норме пространства $L^{p^{\prime}}(Q)$. Для этого используем теорему Витали о сходимости в такой форме [16, с. 167]: если $z_{N} \rightarrow z$ п.в. в $Q$ при $N \rightarrow \infty$ и интегралы от функций $\left|z_{N}\right|^{p^{\prime}}$ равностепенно абсолютно непрерьвны, то $z_{N} \rightarrow z$ в норме пространства $L^{p^{\prime}}(Q)$. Запишем первое из неравенств леммы 2.6, подставляя $u_{N}$ вместо $u$ :

$$
\begin{aligned}
& \left(\int_{E}\left|z_{i N}\right|^{p^{\prime}} d Q\right)^{1 / p^{\prime}}=\left(\int_{E}\left|A_{i}\left(t, x, u_{N}, D v\right)\right|^{p^{\prime}} d Q\right)^{1 / p^{\prime}} \\
& \quad \leqslant C\left(\|D v\|_{L^{p}(E)}^{p-1}+\delta\left\|u_{N}\right\|_{X}^{q / p^{\prime}}+\delta\left\|u_{N}\right\|_{X}^{r / p^{\prime}}+C(\delta)\left\|h_{0}\right\|_{L^{p^{\prime}(E)}}\right) .
\end{aligned}
$$

По условию последовательность $\left\|u_{N}\right\|_{X}$ ограничена, поэтому по заданному $\varepsilon>0$ можно подобрать такое $\delta>0$, чтобы выполнялись неравенства: $\delta\left\|u_{N}\right\|_{X}^{q / p^{\prime}}<\varepsilon, \delta\left\|u_{N}\right\|_{X}^{r / p^{\prime}}<\varepsilon$. По выбранному $\delta$ подберем $\delta_{1}$ так, чтобы для любого измеримого множества $E \subset Q$, удовлетворяюшего условию mes $E<\delta_{1}$, вьполнялись неравенства: $\|D v\|_{L^{p}(E)}^{p-1}<\varepsilon ; C(\delta)\left\|h_{0}\right\|_{L^{p^{\prime}(E)}}<\varepsilon$. Это возможно, так как по условию $D v \in L^{p}(Q)$ и $h_{0} \in L^{p^{\prime}}(Q)$, следовательно, функции $|D v|^{p}$ и $\left|h_{0}\right|^{p^{\prime}}$ имеют абсолютно непрерывные интегралы. Подставляя приведенные неравенства в формулу (2.6), получим оценку $\int_{E}\left|z_{i N}\right|^{p^{\prime}} d Q \leqslant(4 C \varepsilon)^{p^{\prime}}$, где постоянная $C$ не зависит от номера $N$. Это значит, что функции $\left|z_{i N}\right|^{p^{\prime}}$ имеют равностепенно абсолютно непрерывные интегралы. Тем самым выполнены условия теоремы Витали для последовательности $z_{i N}$, согласно которой $z_{i N} \rightarrow z_{i}$ при $N \rightarrow \infty$ в норме пространства $L^{p^{\prime}}(Q)$. Непрерывность оператора $a_{i}(u, v)$ вытекает из того, что он составлен как композиция операторов дифференцирования и оператора суперпозиции, поэтому его непрерывность есть следствие его ограниченности. В свою очередь, ограниченность оператора $a_{i}(u, v)$ следует из леммы 2.6 , примененной к множеству $E=Q$. Это завершает доказательство леммы.

Согласно лемме 2.7 последовательность $w_{0 N}$, определенная равенством (2.5) для случая $i=0$, слабо сходится к некоторой функции $w_{0}$ в пространстве $L^{q^{\prime}}(Q)$. Эта последовательность обладает дополнительными свойствами, которые указываются в следуюшем утверждении.

Лемма 2.9. Пусть $\left\|u_{N}\right\|_{X} \leqslant$. Т. Тогда последовательность $\left|w_{0 N}\right|^{q^{\prime}}$ имеет равностепенно абсолютно непрерьвнье интеграль. Если дополнительно известно, что $u_{N} \rightarrow u, D u_{N} \rightarrow D$ и почти всюду в $Q$, то предельный әлемент $w_{0}=A_{0}(t, x, u, D u)$.

ДокАЗАТЕЛЬСтво. Применим лемму 2.6 для случая $i=0$, подставляя $u_{N}$ вместо $u$, что дает

$$
\begin{aligned}
& \left(\int_{E}\left|w_{0 N}\right|^{q^{\prime}} d Q\right)^{1 / q^{\prime}}=\left(\int_{E}\left|A_{0}\left(t, x, u_{N}, D u_{N}\right)\right|^{q^{\prime}} d Q\right)^{1 / q^{\prime}} \\
& \quad \leqslant C\left[\delta\left(\left\|u_{N}\right\|_{X}^{p / q^{\prime}}+\left\|u_{N}\right\|_{X}^{q-1}+\left\|u_{N}\right\|_{X}^{r / p^{\prime}}\right)+C_{1}(\delta)\left\|h_{2}\right\|_{L^{q^{\prime}}(E)}\right] .
\end{aligned}
$$


По условию $\left\|u_{N}\right\|_{X} \leqslant C$, поэтому слагаемое, содержащее множитель $\delta$, может быть сделано меньше $\varepsilon$ для любого заданного $\varepsilon>0$ за счет выбора малого $\delta>0$. При выбранном таким способом $\delta$ последнее слагаемое в формуле (2.7) может быть сделано меньше $\varepsilon$ за счет выбора множества $E$ малой меры. В результате неравенство (2.7) примет вид $\int_{E}\left|w_{0 N}\right|^{q^{\prime}} d Q \leqslant(2 C \varepsilon)^{q^{\prime}}$, где постоянная $C$ не зависит от $N=1,2, \ldots$ Это значит, что последовательность $\left|w_{0 N}\right|^{q^{\prime}}$ имеет равностепенно абсолютно непрерывные интегралы. Если при этом известно, что $u_{N} \rightarrow u$, $D u_{N} \rightarrow D u$ почти всюду в $Q$, то $w_{0 N} \rightarrow A_{0}(t, x, u, D u)$ почти всюду в $Q$, следовательно, выполнены условия теоремы Витали о сходимости, согласно которой $w_{0 N} \rightarrow A_{0}(t, x, u, D u)$ в норме пространства $L^{q^{\prime}}(Q)$. По лемме 2.7 одновременно $w_{0 N} \rightarrow w_{0}$ слабо в $L^{q^{\prime}}(Q)$. Из сравнения приведенных сходимостей следует, что $w_{0}=A_{0}(t, x, u, D u)$. Это завершает доказательство леммы.

\section{§3. Конечномерные приближения}

Для доказательства разрешимости рассматриваемой задачи применим метод Фаэдо-Галёркина. Так как функция $\beta(u)$ может обратиться в нуль при $u=0$, то введем функцию $\beta_{N}(u)=\beta(u)+\frac{1}{N}$, выбирая в качестве $N$ натуральные числа.

В пространстве $C_{0}^{1}(\bar{\Omega})$ выделим полную счетную систему линейно независимых функций $e_{1}(x), e_{2}(x), \ldots$. Заметим, что эта система будет полной в каждом из пространств $L^{r}(\Omega)$ и $\stackrel{\circ}{W} \underset{p}{1}(\Omega)$. Ишем приближенњые решения уравнения $(0.1)$ в виде $u_{N}=\sum_{k=1}^{N} c_{N k}(t) e_{k}(x)$, и для каждого натурального $N=1,2, \ldots$ составим систему обыкновенных дифференциальных уравнений

$$
\begin{aligned}
\left(\left(\beta\left(u_{N}\right)+\frac{1}{N}\right) u_{N}^{\prime}, e_{m}\right)+\sum_{i=1}^{n}\left(A_{i}\left(t, x, u_{N}, D u_{N}\right), D_{i} e_{m}\right) & \\
+\left(A_{0}\left(t, x, u_{N}, D u_{N}\right), e_{m}\right) & =0 \quad(m=1,2, \ldots, N)
\end{aligned}
$$

которую дополним начальным условием $u_{N}(0, x)=u_{0 N}$. Эти условия выбираются таким образом, чтобы функции $u_{N}(0, x)=\sum_{k=1}^{N} c_{N k}(0) e_{k}(x)$ сходились к заданной функции $u_{0}(x)$ в норме пространства $L^{r}(\Omega)$. Убедимся, что система $(3.1)$ локально разрешима. Запишем первое слагаемое системы в развернутом виде

$$
\begin{aligned}
& \sum_{k=1}^{N}\left(\left(\beta\left(u_{N}\right)+\frac{1}{N}\right) e_{k}, e_{m}\right) c_{N k}^{\prime}(t) \\
& =\sum_{k=1}^{N}\left(\sqrt{\beta\left(u_{N}\right)+\frac{1}{N}} e_{k}, \sqrt{\beta\left(u_{N}\right)+\frac{1}{N}} e_{m}\right) c_{N k}^{\prime}(t) .
\end{aligned}
$$

Отсюда непосредственно видно, что из коэффициентов при $c_{N k}^{\prime}(t)$ получается определитель Грама системы функций $\left(\beta\left(u_{N}\right)+\frac{1}{N}\right)^{1 / 2} e_{k}$, которая линейно независима вместе с исходной системой $e_{k}(k=1,2, \ldots, N)$, так как $\beta\left(u_{N}\right)+\frac{1}{N} \geqslant$ 
$\frac{1}{N}>0$. Это значит, что систему дифференциальных уравнений (3.1) можно привести к нормальной форме невырожденным преобразованием, следовательно, эта система разрешима при каждом $N=1,2, \ldots$ по крайней мере на некотором интервале $\left[0, T_{N}\right)$. Чтобы продолжить решение на весь отрезок $[0, T]$, получим оценку решений $u_{N}$.

При фиксированном $N$ каждое уравнение системы (3.1) под номером $m=1, \ldots, N$ умножим на функцию $c_{N m}(t)$, сложим все уравнения и результат проинтегрируем по отрезку $[0, t]$, считая $t<T_{N}$. В результате получим тождество

$$
\begin{aligned}
& \int_{0}^{t}\left(\left(\beta\left(u_{N}\right)+\frac{1}{N}\right) u_{N}^{\prime}, u_{N}\right) d t \\
& +\sum_{i=1}^{n} \int_{0}^{t}\left(A_{i}\left(t, x, u_{N}, D u_{N}\right), D_{i} u_{N}\right) d t+\int_{0}^{t}\left(A_{0}\left(t, x, u_{N}, D u_{N}\right), u_{N}\right) d t=0
\end{aligned}
$$

которое перепишем в условных обозначениях

$$
J_{1}+J_{2}+J_{3}=0
$$

Оценим каждое слагаемое. Используя функцию $\Gamma(u)=\int_{0}^{u} s \beta(s) d s$, можем записать

$$
\begin{aligned}
\int_{0}^{t}\left(\beta\left(u_{N}\right) u_{N}^{\prime}, u_{N}\right) d t & =\int_{\Omega}\left(\int_{u_{N}(0, x)}^{u_{N}(t, x)} u \beta(u) d u\right) d x \\
& =\int_{\Omega} \Gamma\left(u_{N}(t, x)\right) d x-\int_{\Omega} \Gamma\left(u_{N}(0, x)\right) d x
\end{aligned}
$$

Отсюда

$$
\begin{aligned}
J_{1}=\int_{\Omega} \Gamma\left(u_{N}(t, x)\right) d x-\int_{\Omega} \Gamma\left(u_{N}(0, x)\right) d x & \\
& \quad+\frac{1}{2 N} \int_{\Omega} u_{N}^{2}(t, x) d x-\frac{1}{2 N} \int_{\Omega} u_{N}^{2}(0, x) d x .
\end{aligned}
$$

Согласно оценкам леммы 2.2

$$
\int_{\Omega} \Gamma\left(u_{N}(t, x)\right) d x \geqslant c \int_{\Omega}\left|u_{N}(t, x)\right|^{r} d x=c\left\|u_{N}(t, x)\right\|_{L^{r}(\Omega)}^{r} .
$$

Аналогично,

$$
\int_{\Omega} \Gamma\left(u_{N}(0, x)\right) d x \leqslant C \int_{\Omega}\left(\left|u_{N}(0, x)\right|^{r}+1\right) d x \leqslant C_{1} .
$$

Здесь использован тот факт, что последовательность $u_{N}(0, x)$ согласно построению сходится к функции $u_{0}(x)$ в пространстве $L^{r}(\Omega)$, следовательно, нормы этих функций в указанном пространстве равномерно ограничены. По условию степень 
$r \geqslant 2$, так что пространство $L^{r}(\Omega)$ непрерывно вложено в $L^{2}(\Omega)$. Это значит, что $\left\|u_{N}(0, x)\right\|_{L^{2}(\Omega)} \leqslant C\left\|u_{N}(0, x)\right\|_{L^{r}(\Omega)}$, так что интегралы $\int_{\Omega} u_{N}^{2}(0, x) d x$ также равномерно ограничены. В целом для слагаемого $J_{1}$ получается такая оценка

$$
J_{1} \geqslant c\left\|u_{N}(t, x)\right\|_{L^{r}(\Omega)}^{r}-C .
$$

Для оценки суммы $J_{2}+J_{3}$ воспользуемся условием коэрцитивности $3 \mathrm{~A}$, что дает

$$
\begin{aligned}
J_{2}+J_{3} \geqslant & c_{0} \int_{0}^{t} \int_{\Omega}\left|D u_{N}\right|^{p} d x d t-\delta \int_{0}^{t} \int_{\Omega} g(x)\left|u_{N}\right|^{p} d x d t \\
& -\delta \int_{0}^{t} \int_{\Omega} g_{1}(t)\left|u_{N}\right|^{r} d x d t-C_{2}(\delta) \int_{0}^{t} \int_{\Omega} g_{2}(t, x) d x d t .
\end{aligned}
$$

Оценим слагаемые с множителем $\delta$. Для определенности рассмотрим случай $p<n$, так что функция $g(x) \in L^{s}(\Omega)$, где $s=\frac{n}{p}$. По неравенству Гёльдера с показателями $s$ и $s^{\prime}$ имеем

$$
\begin{aligned}
\int_{\Omega} g(x)\left|u_{N}\right|^{p} d x d t & \leqslant\left(\int_{\Omega}|g(x)|^{s} d x\right)^{1 / s}\left(\int_{\Omega}\left|u_{N}\right|^{p s^{\prime}} d x\right)^{1 / s^{\prime}} \\
& =\|g\|_{L^{s}(\Omega)}\left\|u_{N}\right\|_{L^{p s^{\prime}}(\Omega)}^{p} \\
& \leqslant C\|g\|_{L^{s}(\Omega)}\left\|u_{N}\right\|_{W_{p}^{1}(\Omega)}^{p}=C\|g\|_{L^{s}(\Omega)}\left\|D u_{N}\right\|_{L^{p}(\Omega)}^{p} .
\end{aligned}
$$

Здесь использован тот факт, что $p s^{\prime}=\frac{n p}{n-p}$, следовательно, по теоремам вложения пространство $\stackrel{\circ}{W} \underset{p}{1}(\Omega)$ непрерьвно вложено в пространство $L^{p s^{\prime}}(\Omega)$. В результате можно оценить первое слагаемое в сумме (3.4), содержащее множитель $\delta$ :

$$
\int_{0}^{t} \int_{\Omega} g(x)\left|u_{N}\right|^{p} d x d t \leqslant C\|g\|_{L^{s}(\Omega)}\left\|D u_{N}\right\|_{L^{p}\left(Q_{t}\right)}^{p}
$$

Здесь введено обозначение $Q_{t}=(0, t) \times \Omega$. Аналогично рассматриваются случаи $p=n$ и $p>n$.

Перейдем ко второму слагаемому, содержашему множитель $\delta$ в сумме (3.4):

$$
\begin{aligned}
\int_{0}^{t} g_{1}(t)\left(\int_{\Omega}\left|u_{N}\right|^{r} d x\right) d t & =\int_{0}^{t} g_{1}(t)\left\|u_{N}(t, x)\right\|_{L^{r}(\Omega)}^{r} d t \\
& \leqslant \sup _{t}\left\|u_{N}(t, x)\right\|_{L^{r}(\Omega)}^{r} \int_{0}^{t} g_{1}(t) d t \\
& \leqslant\left\|g_{1}\right\|_{L^{1}(0, T)} \sup \left\|u_{N}(t, x)\right\|_{L^{r}(\Omega)}^{r} .
\end{aligned}
$$

В целом для суммы (3.4) получаем такую оценку

$$
\begin{aligned}
J_{2}+J_{3} \geqslant c_{0}\left\|D u_{N}\right\|_{L^{p}\left(Q_{t}\right)}^{p}-\delta C\|g\|_{L^{s}(\Omega)}\left\|D u_{N}\right\|_{L^{p}\left(Q_{t}\right)}^{p} & \\
& -\delta\left\|g_{1}\right\|_{L^{1}(0, T)} \sup _{t}\left\|u_{N}(t, x)\right\|_{L^{r}(\Omega)}^{r}-C_{2}(\delta)\left\|g_{2}\right\|_{L^{1}(Q)} .
\end{aligned}
$$


Объединяя ее с неравенством (3.3) для $J_{1}$ и подставляя в тождество $(3.2)$, получим

$$
\begin{aligned}
c\left\|u_{N}(t, x)\right\|_{L^{r}(\Omega)}^{r} & +c_{0}\left\|D u_{N}\right\|_{L^{p}\left(Q_{t}\right)}^{p} \\
& \leqslant C\left(1+\delta\left\|D u_{N}\right\|_{L^{p}\left(Q_{t}\right)}^{p}+\delta \sup _{t}\left\|u_{N}(t, x)\right\|_{L^{r}(\Omega)}^{r}+C_{2}(\delta)\right) .
\end{aligned}
$$

Ясно, что выбором достаточно малого параметра $\delta>0$ можно добиться следующей оценки

$$
\sup _{t}\left\|u_{N}(t, x)\right\|_{L^{r}(\Omega)}^{r}+\left\|D u_{N}\right\|_{L^{p}\left(Q_{t}\right)}^{p} \leqslant C
$$

где постоянная $C$ не зависит от номера $N$. Отсюда следует, что решения $u_{N}(t, x)$ можно продолжить на весь отрезок $[0, T]$ для каждого номера $N=1,2, \ldots$, и при этом сохраняется приведенная оценка, которую удобно представить в виде

$$
\sup _{t}\left\|u_{N}(t, x)\right\|_{L^{r}(\Omega)} \leqslant C ; \quad\left\|D u_{N}\right\|_{L^{p}(Q)} \leqslant C
$$

что равносильно оценке $\left\|u_{N}\right\|_{X} \leqslant C$. По лемме 2.5 пространство $X$ непрерывно вложено в пространство $L^{q}(Q)$, следовательно, $\left\|u_{N}\right\|_{L^{q}(Q)} \leqslant C$. Объединяя эти оценки с леммой 2.7 , приходим к следующему заключению.

Лемма 3.1. Для кажсдого $N=1,2, \ldots$ система дифференциальных уравнений (3.1) имеет решение $u_{N}(t, x)$, которое определено на отрезке $[0, T] u$ удовлетворяет оценкам:

(a) $\sup _{t}\left\|u_{N}(t, x)\right\|_{L^{r}(\Omega)} \leqslant C ;\left\|D u_{N}\right\|_{L^{p}(Q)} \leqslant C ;$

(b) $\left\|u_{N}\right\|_{X} \leqslant C ;\left\|u_{N}\right\|_{L^{q}(Q)} \leqslant C$;

(c) $\left\|A_{i}\left(t, x, u_{N}, D u_{N}\right)\right\|_{L^{p^{\prime}}(Q)} \leqslant C \quad(i=1, \ldots, n)$;

(d) $\left\|A_{0}\left(t, x, u_{N}, D u_{N}\right)\right\|_{L^{q^{\prime}}(Q)} \leqslant C$.

Здесь постоянные не зависят от номера $N$.

Дальнейшее изложение посвящено изучению свойств последовательности $u_{N}$, а также построенной по ней последовательности $U_{N}=B\left(u_{N}\right)+\frac{1}{N} u_{N}$. Введем конечномерные пространства $E_{N}$, натянутые на первые элементы базиса $e_{1}(x), \ldots, e_{N}(x)$, и пусть $E_{0}=\bigcup_{N=1}^{\infty} E_{N}$. Заметим, что $U_{N}^{\prime}=\left(\beta\left(u_{N}\right)+\frac{1}{N}\right) u_{N}^{\prime}$, поэтому для каждого элемента $e \in E_{N}$ систему приближенных уравнений (3.1) можно представить в таком виде:

$$
\left(U_{N}^{\prime}, e\right)+\sum_{i=1}^{n}\left(A_{i}\left(t, x, u_{N}, D u_{N}\right), D_{i} e\right)+\left(A_{0}\left(t, x, u_{N}, D u_{N}\right), e\right)=0
$$

ЛЕмма 3.2. Для последовательности $U_{N}$ справедливо неравенство $\sup _{t}\left\|U_{N}(t, x)\right\|_{L^{r^{\prime}}(\Omega)} \leqslant C$, где постоянная не зависит от номера $N=1,2, \ldots$. 
ДоКАЗАТЕЛЬСТВо. Используя оценку $|B(u)| \leqslant C\left(|u|^{r-1}+1\right)$ из леммы 2.1 (a), найдем, что $|B(u)|^{r^{\prime}} \leqslant C\left(|u|^{r}+1\right)$. Так как $\left|U_{N}\right|^{r^{\prime}} \leqslant C\left(\left|B\left(u_{N}\right)\right|^{r^{\prime}}+\left|N^{-1} u_{N}\right|^{r^{\prime}}\right)$, то

$$
\begin{aligned}
\left\|U_{N}(t, x)\right\|_{L^{r^{\prime}(\Omega)}}^{r^{\prime}} & =\int_{\Omega}\left|U_{N}(t, x)\right|^{r^{\prime}} d x \\
& \leqslant C\left(\int_{\Omega}\left|u_{N}\right|^{r} d x+\int_{\Omega}\left|N^{-1} u_{N}\right|^{r^{\prime}} d x+\operatorname{mes} \Omega\right) .
\end{aligned}
$$

Отсюда

$$
\sup _{t}\left\|U_{N}(t, x)\right\|_{L^{r^{\prime}}(\Omega)}^{r^{\prime}} \leqslant C\left(\sup _{t}\left\|u_{N}\right\|_{L^{r}(\Omega)}^{r}+\sup _{t}\left\|N^{-1} u_{N}\right\|_{L^{r^{\prime}}(\Omega)}^{r^{\prime}}+\operatorname{mes} \Omega\right) .
$$

Так как $r \geqslant 2$, то $r^{\prime} \leqslant 2$, и потому $\left\|N^{-1} u_{N}\right\|_{L^{r^{\prime}}(\Omega)} \leqslant C\left\|N^{-1} u_{N}\right\|_{L^{r}(\Omega)}$. Применив лемму 3.1 , получим утверждение.

Лемма 3.3. Пусть $t, t+h \in[0, T]$. Тогда для каждой функиии е $\in E_{N}$ справедлива оценка, в которой постоянная $C$ не зависит от $N$ :

$\left|\left(\Delta_{h} U_{N}, e\right)\right| \equiv\left|\left(U_{N}(t+h, x)-U_{N}(t, x), e\right)\right| \leqslant C\left(|h|^{1 / p}\|D e\|_{L^{p}(\Omega)}+|h|^{1 / q}\|e\|_{L^{q}(\Omega)}\right)$.

ДокАЗАТЕЛЬство. Представим рассматриваемое прирашение по формуле Ньютона-Лейбница

$$
\left(\Delta_{h} U_{N}, e\right)=\int_{t}^{t+h}\left(U_{N}^{\prime}(\tau, x), e\right) d \tau
$$

Так как $e \in E_{N}$, то можно воспользоваться системой (3.5), что дает тождество

$$
\begin{aligned}
\left(\Delta_{h} U_{N}, e\right)=-\sum_{i=1}^{n} \int_{t}^{t+h}\left(A_{i}\left(\tau, x, u_{N}, D u_{N}\right), D_{i} e\right) d \tau & \\
& -\int_{t}^{t+h}\left(A_{0}\left(\tau, x, u_{N}, D u_{N}\right), e\right) d \tau .
\end{aligned}
$$

Отсюда

$$
\begin{aligned}
\left|\left(\Delta_{h} U_{N}, e\right)\right| \leqslant & \sum_{i=1}^{n}\left(\int_{t}^{t+h}\left\|A_{i}\left(\tau, x, u_{N}, D u_{N}\right)\right\|_{L^{p^{\prime}}(\Omega)}^{p^{\prime}} d \tau\right)^{1 / p^{\prime}}\left(\int_{t}^{t+h}\left\|D_{i} e\right\|_{L^{p}(\Omega)}^{p} d \tau\right)^{1 / p} \\
& +\left(\int_{t}^{t+h}\left\|A_{0}\left(\tau, x, u_{N}, D u_{N}\right)\right\|_{L^{q^{\prime}}(\Omega)}^{q^{\prime}} d \tau\right)^{1 / q^{\prime}}\left(\int_{t}^{t+h}\|e\|_{L^{q}(\Omega)}^{q} d \tau\right)^{1 / q}
\end{aligned}
$$

Учтем, что функция $e(x)$ не зависит от переменной $\tau \in[0, T]$, поэтому

$$
\begin{aligned}
\left|\left(\Delta_{h} U_{N}, e\right)\right| \leqslant \sum_{i=1}^{n}|h|^{1 / p}\left\|D_{i} e\right\|_{L^{p}(\Omega)}\left\|A_{i}\left(\tau, x, u_{N}, D u_{N}\right)\right\|_{L^{p^{\prime}}(Q)} & \\
& +|h|^{1 / q}\|e\|_{L^{q}(\Omega)}\left\|A_{0}\left(\tau, x, u_{N}, D u_{N}\right)\right\|_{L^{q^{\prime}}(Q)} .
\end{aligned}
$$

Используя оценку леммы 3.1 , получим утверждение доказываемой леммы. 
ЛЕмма 3.4. Пусть $h$ - фиксированное число, причем $|h| \leqslant 1$. Введем функцию $u_{N}(t+h, x)$, считая ее продолженной нулем вне области $Q$. Тогда

$$
\left\|u_{N}(t+h, x)-u_{N}(t, x)\right\|_{L^{r}(Q)} \leqslant C|h|^{\alpha}, \quad \alpha=\frac{1}{r} \min \left(\frac{1}{p}, \frac{1}{q}\right),
$$

где постоянная $C$ не зависит от $h$ и номера $N$.

ДокАЗАТЕЛЬСТво. Введем функцию $B_{N}(u)=B(u)+N^{-1} u$, и для произвольных $u, v \in \mathbb{R}^{1}$ рассмотрим произведение

$$
\begin{aligned}
\left(B_{N}(u)-B_{N}(v)\right)(u-v) & =(B(u)-B(v))(u-v)+N^{-1}(u-v)^{2} \\
& \geqslant(B(u)-B(v))(u-v) \geqslant c|u-v|^{r} .
\end{aligned}
$$

Здесь использована лемма 2.1(b). Применим это неравенство, полагая $v=$ $u_{N}(t, x), u=u_{N}(t+h, x)$. При этом согласно принятым обозначениям $B_{N}(u)=$ $U_{N}(t+h, x), B_{N}(v)=U_{N}(t, x)$. В результате получим: $c\left|\Delta_{h} u_{N}\right|^{r} \leqslant \Delta_{h} U_{N} \cdot \Delta_{h} u_{N}$. Отсюда

$$
c \int_{\Omega}\left|\Delta_{h} u_{N}\right|^{r} d x \leqslant\left(\Delta_{h} U_{N}, \Delta_{h} u_{N}\right) .
$$

При каждом $t \in[0, T] \Delta_{h} u_{N} \in E_{N}$ согласно построению, поэтому можно применить лемму 3.3 , полагая $e=\Delta_{h} u_{N}$, при условии, что $t+h \in[0, T]$. В результате получим оценку

$$
c \int_{\Omega}\left|\Delta_{h} u_{N}\right|^{r} d x \leqslant C\left(|h|^{1 / p}\left\|D \Delta_{h} u_{N}\right\|_{L^{p}(\Omega)}+|h|^{1 / p}\left\|\Delta_{h} u_{N}\right\|_{L^{q}(\Omega)}\right) .
$$

Для определенности допустим, что $h>0$. Тогда условие $t+h \in[0, T]$ вьполнено, если $t \in[0, T-h]$. Для таких $t$ приведенное выше неравенство справедливо и его можно интегрировать по отрезку $[0, T-h]$, что дает

$$
c \int_{0}^{T-h} \int_{\Omega}\left|\Delta_{h} u_{N}\right|^{r} d x d t \leqslant C\left(|h|^{1 / p}\left\|D u_{N}\right\|_{L^{p}(Q)}+|h|^{1 / q}\left\|u_{N}\right\|_{L^{q}(Q)}\right) .
$$

Используя еще лемму 3.1 , получим

$$
\int_{0}^{T-h} \int_{\Omega}\left|\Delta_{h} u_{N}\right|^{r} d x d t \leqslant C|h|^{\gamma}, \quad \gamma=\min \left(\frac{1}{p}, \frac{1}{q}\right) .
$$

Пусть теперь $t \in(T-h, T)$. Тогда в соответствии с договоренностью $u_{N}(t+h, x)=0$, так что $\Delta_{h} u_{N}=-u_{N}(t, x)$. Отсюда для $|h| \leqslant 1$

$$
\begin{aligned}
\int_{T-h}^{T} \int_{\Omega}\left|\Delta_{h} u_{N}\right|^{r} d x d t & =\int_{T-h}^{T}\left\|u_{N}(t, x)\right\|_{L^{r}(\Omega)}^{r} d t \\
& \leqslant h \sup _{t}\left\|u_{N}(t, x)\right\|_{L^{r}(\Omega)}^{r} \leqslant C h \leqslant C h^{\gamma} .
\end{aligned}
$$

Здесь вновь использована лемма 3.1. Складывая оценки (3.7) и (3.8), получим утверждение леммы. 
ЛЕМма 3.5. Из последовательности $u_{N}$ можсно извлечь подпоследовательность, снова обозначаемую $u_{N}$, такую, что для некоторой функиии $u(t, x)$ справедливы следующие предельные соотношения при $N \rightarrow \infty$ :

(a) $\left\|u_{N}-u\right\|_{L^{m}(Q)} \rightarrow 0, m=\min (p, r)$;

(b) $u_{N}(t, x) \rightarrow u(t, x)$ n.в. в $Q$;

(c) $U_{N}(t, x) \rightarrow U(t, x)=B(u(t, x))$ n.в. $в Q$.

ДокАЗАТЕЛьство. Используем теорему Рисса о компактности в пространстве $L^{m}$ в следующей формулировке [17, с. 278]. Пусть $g \subset \mathbb{R}^{n}$ - ограниченная область пространства $\mathbb{R}^{n}$. Множество $M$ функций $f \in L^{m}(g)$ компактно в $L^{m}(g)$, $1 \leqslant m<\infty$, если: 1 ) оно ограничено в $\left.L^{m}(g), 2\right)$ равностепенно непрерывно по сдвигу в $L^{m}(g)$, т.е.

$$
\sup _{f \in M ;|h| \leqslant \delta}\|f(x+h)-f(x)\|_{L^{m}(g)} \rightarrow 0 \quad(\delta \rightarrow 0) .
$$

При этом предполагается, что $f=0$ вне области $g$.

Полагаем $g=Q$. Множество $M$ состоит из функций $u_{N}(N=1,2, \ldots)$. По лемме 3.1 (a) $\left\|u_{N}\right\|_{L^{\infty}\left(0, T ; L^{r}(\Omega)\right)} \leqslant C$. Отсюда следует благодаря выбору $m=\min (p, r)$, что $\left\|u_{N}\right\|_{L^{m}(Q)} \leqslant C$, так что первое условие теоремы о компактности выполнено. Проверим второе. Фиксируем число $\delta>0$ и пусть $|h| \leqslant \delta$. Сдвиги функций $u_{N}$ по переменной $t$ уже оценены в лемме 3.4. При этом оценка получена в норме пространства $L^{r}(Q)$. Так как $m \leqslant r$, то упомянутую оценку можно использовать в такой форме

$$
\begin{gathered}
\left\|u_{N}(t+h, x)-u_{N}(t, x)\right\|_{L^{m}(Q)}=\left\|\Delta_{h} u_{N}\right\|_{L^{m}(Q)} \leqslant C \delta^{\alpha} \\
\alpha=\min \left(\frac{1}{r p}, \frac{1}{r q}\right) .
\end{gathered}
$$

Перейдем к сдвигам по переменной $x \in \Omega$. Рассмотрим подробно прирашение по переменной $x_{1}$, для чего используем представление $x=\left(x_{1}, x^{\prime}\right)$. Допустим, что отрезок $\left[x_{1}, x_{1}+h_{1}\right]$ при заданном $x^{\prime}$ полностью содержится в области $\Omega$. Тогда

$$
\Delta_{h_{1}} u_{N}=u_{N}\left(t, x_{1}+h_{1}, x^{\prime}\right)-u_{N}\left(t, x_{1}, x^{\prime}\right)=\int_{x_{1}}^{x_{1}+h_{1}} D_{1} u_{N}\left(t, y, x^{\prime}\right) d y,
$$

так что

$$
\begin{aligned}
\left|\Delta_{h_{1}} u_{N}\right| & \leqslant\left(\int_{x_{1}}^{x_{1}+h_{1}}\left|D_{1} u_{N}\right|^{p} d y\right)^{1 / p}\left(\int_{x_{1}}^{x_{1}+h_{1}} d y\right)^{1 / p^{\prime}} \\
& \leqslant\left|h_{1}\right|^{1 / p^{\prime}}\left(\int_{\Omega_{1}\left(x^{\prime}\right)}\left|D_{1} u_{N}\right|^{p} d y\right)^{1 / p}
\end{aligned}
$$

Здесь $\Omega_{1}\left(x^{\prime}\right)$ - сечение области $\Omega$ прямой $l\left(x^{\prime}\right)$, которая получается фиксированием точки $x^{\prime}$ и изменением переменной $x_{1} \in \mathbb{R}^{1}$. Если же точка $\left(x_{1}+h_{1}, x^{\prime}\right)$ выходит 
за пределы области $\Omega$, то в соответствии с договоренностью $u_{N}\left(t, x_{1}+h_{1}, x^{\prime}\right)=0$, так что $\Delta_{h_{1}} u_{N} \equiv-u_{N}\left(t, x_{1}, x^{\prime}\right)$. Однако в этом случае на интервале $x_{1}<y<$ $x_{1}+h_{1}$ прямой $l\left(x^{\prime}\right)$ найдется точка $\left(x_{1}^{0}, x^{\prime}\right)$, лежащая на границе $S$ области $\Omega$. Так как $\left.u\right|_{S}=0$, то можно записать такое тождество: $\Delta_{h_{1}} u_{N}=u_{N}\left(t, x_{1}^{0}, x^{\prime}\right)-$ $u_{N}\left(t, x_{1}, x^{\prime}\right)$, причем $\left|x_{1}-x_{1}^{0}\right|<h_{1}$. Мы снова оказались в условиях применимости формулы (3.10), откуда следует, что во всех возможных случаях верно неравенство

$$
\left|\Delta_{h_{1}} u_{N}\right| \leqslant\left|h_{1}\right|^{1 / p^{\prime}}\left(\int_{\Omega_{1}\left(x^{\prime}\right)}\left|D_{1} u_{N}\right|^{p} d y\right)^{1 / p}
$$

Из него достаточно очевидным образом вытекает оценка

$$
\int_{Q}\left|\Delta_{h_{1}} u_{N}\right|^{p} d Q \leqslant C\left|h_{1}\right|^{p / p^{\prime}} \int_{Q}\left|D_{1} u_{N}\right|^{p} d Q
$$

Так как $\left\|D u_{N}\right\|_{L^{p}(Q)} \leqslant C$ согласно лемме 3.1 , то

$$
\left\|\Delta_{h_{1}} u_{N}\right\|_{L^{p}(Q)} \leqslant C\left|h_{1}\right|^{1 / p^{\prime}}
$$

Ясно, что эта оценка справедлива для любой переменной $x_{1}, \ldots, x_{n}$, так что

$$
\left\|\Delta_{h_{i}} u_{N}\right\|_{L^{p}(Q)} \leqslant C\left|h_{i}\right|^{1 / p^{\prime}} \quad(i=1, \ldots, n)
$$

В случае совместного сдвига на вектор $\bar{h} \in \mathbb{R}^{n}$, где $|\bar{h}| \leqslant \delta$, представим разность $\Delta_{\bar{h}} u_{N}$ как сумму сдвигов по каждой переменной $x_{1}, \ldots, x_{n}$ и воспользуемся формулой (3.11), что приведет к неравенству $\left\|\Delta_{\bar{h}} u_{N}\right\|_{L^{p}(Q)} \leqslant C \delta^{1 / p^{\prime}}$, откуда следует благодаря выбору $m$, что

$$
\left\|\Delta_{\bar{h}} u_{N}\right\|_{L^{m}(Q)} \leqslant C \delta^{1 / p^{\prime}}
$$

Объединяя этот результат с оценкой (3.9), при совместном сдвиге по переменньп $(t, x) \in Q$ получим

$$
\left\|u_{N}(t+h, x+\bar{h})-u_{N}(t, x)\right\|_{L^{m}(Q)} \leqslant C \delta^{\rho}, \quad \rho=\min \left(\alpha, \frac{1}{p^{\prime}}\right) .
$$

Здесь $|h|,|\bar{h}| \leqslant \delta$, постоянная $C$ не зависит от $\delta$ и $N$. Тем самым выполнено и второе условие теоремы о компактности, значит, последовательность $u_{N}$ компактна в пространстве $L^{m}(Q)$. Это доказывает первое утверждение леммы. Второе является его следствием. Проверим последнее. Согласно построению $U_{N}=B\left(u_{N}\right)+N^{-1} u_{N}$. При этом $u_{N} \rightarrow u$ почти всюду в $Q$. Фиксируем точку сходимости $(t, x) \in Q$. Тогда $N^{-1} u_{N}(t, x) \rightarrow 0(N \rightarrow \infty), B\left(u_{N}(t, x)\right) \rightarrow B(u(t, x))$, так что в целом $U_{N}(t, x) \rightarrow B(u(t, x))=U(t, x)$. Это завершает доказательство леммы. 
ЛЕМма 3.6. Из последовательности $u_{N}$ можсно извлечь подпоследовательность, снова обозначаемую $u_{N}$, такую, что при $N \rightarrow \infty$ :

(a) $u_{N} \rightarrow$ сллабо в $X_{1}=L^{p}(0, T ; \stackrel{\circ}{W} \underset{p}{1}(\Omega))$ и при этом $u \in X$;

(b) $A_{i}\left(t, x, u_{N}, D u_{N}\right)=w_{i N} \rightarrow w_{i}$ слабо в $L^{p^{\prime}}(Q), i=1, \ldots, n$; $A_{0}\left(t, x, u_{N}, D u_{N}\right)=w_{0 N} \rightarrow w_{0}$ слабо в $L^{q^{\prime}}(Q)$, где $w_{i}(i=1, \ldots, n)$ - некоторые элементы пространства $L^{p^{\prime}}(Q), w_{0}$ - әлемент пространства $L^{q^{\prime}}(Q)$;

(c) $U_{N} \rightarrow U=B(u)$ *-слабо в $L^{\infty}\left(0, T ; L^{r^{\prime}}(\Omega)\right)$, m.e. $\left\langle U_{N}, w\right\rangle \rightarrow\langle U, w\rangle$ для любого әлемента $w \in L^{1}\left(0, T ; L^{r}(\Omega)\right)$, при этом

$$
\|U(t, x)\|_{L^{\infty}\left(0, T ; L^{r^{\prime}}(\Omega)\right)}<C .
$$

ДокАЗАТЕЛьСтво. Согласно определению нормы в пространстве $X_{1}$ и в силу оценок леммы 3.1 (а) справедливы неравенства $\left\|u_{N}\right\|_{X_{1}}=\left\|D u_{N}\right\|_{L^{p}(Q)} \leqslant C$. Так как пространство $X_{1}$ рефлексивно, то можно считать, что $u_{N} \rightarrow z$ слабо в $X_{1}$. Одновременно по лемме 3.5 (а) $u_{N} \rightarrow u$ в пространстве $L^{m}(Q)$. Из сравнения приведенных сходимостей следует, что $z=u$, так что $u_{N} \rightarrow u$ слабо в $X_{1}$. В частности, $u \in X_{1}$. Из оценок леммы 3.1(a) с очевидностью вытекает, что $\|u\|_{L^{\infty}\left(0, T ; L^{r}(\Omega)\right)} \leqslant C$, т.е. $u \in X_{0}$. А тогда $u \in X=X_{0} \cap X_{1}$. Это доказьвает пункт (a). Аналогично из лемм 3.2 и 3.5 (c) вытекает пункт (c). Наконец, пункт (b) следует из объединения лемм 3.1(b) и 2.7, что завершает доказательство.

Обозначим через $Y_{N}$ пространство функций вида $\sum_{k=1}^{N} d_{k}(t) e_{k}(x)$, где $d_{k} \in C^{1}[0, T]$. И пусть $Y_{0}=\bigcup_{N=1}^{\infty} Y_{N}$. Очевидно, что $Y_{0} \subset X$.

Лемма 3.7. Для функиии $U=B(u)$, построенной в лемме 3.5 , определена производная $U^{\prime}=\frac{\partial}{\partial t} B(u)$ как әлемент пространства $\mathscr{D}^{\prime}\left(0, T ; L^{r^{\prime}}(\Omega)\right)$. Эта производная задает непрерывный линейный функционал на пространстве $X$ и удовлетворяет уравнению

(a) $\left\langle U^{\prime}, v\right\rangle+\sum_{i=1}^{n}\left\langle w_{i}, D_{i} v\right\rangle+\left\langle w_{0}, v\right\rangle=0, v \in X$.

Здесь әлементы $w_{i}(i=0,1, \ldots, n)$ взяты из леммы 3.6. При этом для каждого $v \in Y_{0}$ при $N \rightarrow \infty$

(b) $\left\langle U_{N}^{\prime}, v\right\rangle \rightarrow\left\langle U^{\prime}, v\right\rangle, v \in Y_{0}$.

ДоказАТЕЛЬство. Пусть $\varphi(t) \in \mathscr{D}(0, T), e(x) \in L^{r}(\Omega) . \quad$ Полагаем $w=$ $\varphi^{\prime}(t) e(x)$. Очевидно, что $w \in L^{1}\left(0, T ; L^{r}(\Omega)\right)$, поэтому согласно лемме 3.6(c) $\left\langle U_{N}, \varphi^{\prime} e\right\rangle \rightarrow\left\langle U, \varphi^{\prime} e\right\rangle$ при $N \rightarrow \infty$. Это соотношение означает, что определены производные $U_{N}^{\prime}$ и $U^{\prime}$ как элементы пространства $\mathscr{D}^{\prime}\left(0, T ; L^{r^{\prime}}(\Omega)\right)$, и при этом

$$
\left\langle U_{N}^{\prime}, w\right\rangle \rightarrow\left\langle U^{\prime}, w\right\rangle, \quad w=\varphi(t) e(x), \quad \varphi \in \mathscr{D}(0, T)
$$

Пусть задана функция $v \in Y_{0}$, так что $v=\sum_{k=1}^{M} d_{k}(t) e_{k}(x)$, где $M-$ фиксированное целое число. Считая $N \geqslant M$, запишем приближенные уравнения (3.5) для 
функций $e_{1}(x), \ldots, e_{M}(x)$, умножим уравнение под номером $k=1,2, \ldots, M$ на числовую функцию $d_{k}(t)$ и все результаты сложим. Это дает следуюшее равенство

$$
\left\langle U_{N}^{\prime}, v\right\rangle+\sum_{i=1}^{n}\left\langle w_{i N}, D_{i} v\right\rangle+\left\langle w_{0 N}, v\right\rangle=0, \quad N \geqslant M
$$

Выберем функции $d_{k}(t) \in \mathscr{D}(0, T)$ для $k=1, \ldots, M$. Тогда можно использовать соотношение (3.12), так что предыдущее равенство с учетом предельных соотношений леммы $3.6(\mathrm{~b})$ дает при $N \rightarrow \infty$ тождество

$$
\left\langle U^{\prime}, v\right\rangle+\sum_{i=1}^{n}\left\langle w_{i}, D_{i} v\right\rangle+\left\langle w_{0}, v\right\rangle=0
$$

Оно верно для функций вида $v=\sum_{k=1}^{M} d_{k}(t) e_{k}(x)$, где $d_{k}(t) \in \mathscr{D}(0, T)$. Множество таких функций плотно в пространстве $X$, поэтому из (3.14) следует, что $U^{\prime}=\sum_{i=1}^{n} D_{i} w_{i}-w_{0}$. Для $i=1, \ldots, n w_{i} \in L^{p^{\prime}}(Q)$, поэтому

$$
D_{i} w_{i} \in L^{p^{\prime}}\left(0, T ; W_{p^{\prime}}^{-1}(\Omega)\right)=X_{1}^{*}
$$

Они определяют непрерывные линейные функционалы на пространстве $X_{1}$, следовательно, и на его части $X=X_{0} \cap X_{1}$. Функция $w_{0} \in L^{q^{\prime}}(Q)$ и потому задает непрерывный линейный функционал на пространстве $L^{q}(Q)$, а тогда и на его части $X \subset L^{q}(Q)$, что следует из леммы 2.5. В целом функция $U^{\prime}$ задает непрерывный линейньй функционал на пространстве $X$. В частности, тождество (3.14) продолжается по непрерывности на все функции $v \in X$, что доказывает пункт (а) леммы.

Перейдем к (b). Из (3.12) следует, что требуемое соотношение уже доказано для функций специального вида. Задача состоит в том, чтобы расширить запас множителей. Пусть задана функция $v \in Y_{0}$. Для нее верно тождество (3.13). Одновременно эту функцию можно подставить в тождество (3.14), так как согласно предыдушему оно верно для всех функций $v \in X$. В тождестве (3.13) устремим $N \rightarrow \infty$. При этом все слагаемые, кроме первого, будут стремиться к соответствуюшим слагаемым тождества (3.14), что установлено в лемме 3.6(b). Но тогда первое слагаемое тождества (3.13) также будет стремиться к первому слагаемому тождества (3.14). Это в точности соответствует пункту (b) леммы, что завершает ее доказательство.

ЛЕмма 3.8. Из последовательности $u_{N}$ можно извлечь подпоследовательность, снова обозначаемую $u_{N}$, такую, что при $N \rightarrow \infty$ :

(a) $B\left(u_{N}(T, x)\right) \rightarrow B(u(T, x))$ слабо в $L^{r^{\prime}}(\Omega)$;

(b) $B\left(u_{N}(0, x)\right) \rightarrow B(u(0, x))$ сильно в $L^{r^{\prime}}(\Omega)$;

(c) $B(u(0, x))=B\left(u_{0}(x)\right)$. 
ДоКАЗАТЕЛЬСтво. Сначала убедимся, что последовательность $U_{N}(T, x) \rightarrow$ $U(T, x)$ слабо в пространстве $L^{r^{\prime}}(\Omega)$. Согласно лемме $3.2\left\|U_{N}(T, x)\right\|_{L^{r^{\prime}}(\Omega)} \leqslant C$, поэтому можно считать, что $U_{N}(T, x) \rightarrow W$ слабо в $L^{r^{\prime}}(\Omega)$. Зафиксируем элемент $e(x) \in E_{0}$ и введем функцию $v=t e(x)$. Непосредственньм вычислением находим, что

$$
\left\langle U_{N}^{\prime}, t e\right\rangle=T\left(U_{N}(T, x), e\right)-\left\langle U_{N}, e\right\rangle .
$$

В последнем равенстве устремим $N \rightarrow \infty$. Так как $v=t e \in Y_{0}$, то можно использовать лемму $3.7(\mathrm{~b})$, согласно которой в пределе получим тождество

$$
\left\langle U^{\prime}, t e\right\rangle=T(W, e)-\langle U, e\rangle .
$$

Вычитая его из тождества

$$
\left\langle U^{\prime}, t e\right\rangle=T(U(T, x), e)-\langle U, e\rangle
$$

найдем, что $(U(T, x)-W, e)=0 ; e \in E_{0}$. Так как $E_{0}$ плотно в пространстве $L^{r}(\Omega)$, то $U(T, x)=\stackrel{\circ}{W}$, что в принятых обозначениях $U(T, x)=B(u(T, x))$ приводит к соотношению: $U_{N}(T, x) \rightarrow B(u(T, x))$ слабо в $L^{r^{\prime}}(\Omega)$. Согласно определению $U_{N}=B\left(u_{N}\right)+N^{-1} u_{N}$, так что для элемента $e \in L^{r}(\Omega)$

$$
\left(B\left(u_{N}(T, x)\right), e\right)=\left(U_{N}(T, x), e\right)-\frac{1}{N}\left(u_{N}(T, x), e\right) \rightarrow(B(u(T, x)), e)
$$

при $N \rightarrow \infty$, так как множители $\left(u_{N}(T, x), e\right)$ равномерно ограничены:

$$
\left|\left(u_{N}(T, x), e\right)\right| \leqslant\left\|u_{N}(T, x)\right\|_{L^{r}(\Omega)}\|e\|_{L^{r^{\prime}(\Omega)}} \leqslant C
$$

в силу леммы 3.1 (а). Тем самым доказан пункт (а) леммы. Проверим последующие. Если провести аналогичные вычисления с функцией $v=(T-t) e(x)$, то придем к соотношению: $B\left(u_{N}(0, x)\right) \rightarrow B(u(0, x))$ слабо в $L^{r^{\prime}}(\Omega)$. Убедимся, что сходимость здесь сильная. Для этого используем неравенство (c) леммы 2.1 в такой форме:

$$
\left|B\left(u_{N}(0, x)\right)-B\left(u_{0}(x)\right)\right| \leqslant C\left|u_{N}(0, x)-u_{0}(x)\right|\left(1+\left|u_{N}(0, x)\right|^{r-2}+\left|u_{0}(x)\right|^{r-2}\right) .
$$

Возводя в степень $r^{\prime}$, интегрируя по области $\Omega$ и применяя неравенство Гёльдера с подходящими показателями, получим

$$
\begin{aligned}
& \left\|B\left(u_{N}(0, x)\right)-B\left(u_{0}(x)\right)\right\|_{L^{r^{\prime}}(\Omega)}^{r^{\prime}} \\
& \quad \leqslant C\left\|u_{N}(0, x)-u_{0}(x)\right\|_{L^{r}(\Omega)}^{\frac{1}{r-1}}\left(1+\left\|u_{N}(0, x)\right\|_{L^{r}(\Omega)}^{\frac{r-2}{r-1}}+\left\|u_{0}(x)\right\|_{L^{r}(\Omega)}^{\frac{r-2}{r-1}}\right) .
\end{aligned}
$$

Согласно построению $u_{N}(0, x) \rightarrow u_{0}(x)$ в норме пространства $L^{r}(\Omega)$, а сумма в скобках справа равномерно ограничена. Следовательно, $B\left(u_{N}(0, x)\right) \rightarrow$ $B\left(u_{0}(x)\right)$ в норме пространства $L^{r^{\prime}}(\Omega)$. Сравним это соотношение с установленной выше слабой сходимостью $B\left(u_{N}(0, x)\right)$ к $B(u(0, x))$. Из сравнения следует, что $B(u(0, x))=B\left(u_{0}(x)\right)$ и при этом $B\left(u_{N}(0, x)\right) \rightarrow B(u(0, x))$ в норме пространства $L^{r^{\prime}}(\Omega)$. Тем самым проверены все утверждения леммы, что завершает ее доказательство. 
Лемма 3.9. Функиия $U=B(u(t, x))$ принадлежит $C\left([0, T] ; w-L^{r^{\prime}}(\Omega)\right)$, где символ $w$ - означает слабую топологию пространства $L^{r^{\prime}}(\Omega)$, и при этом как непрерывная функиия она принимает значение $B(u(0, x))=B\left(u_{0}(x)\right)$.

Доказательство. Пусть $t, t+h \in[0, T]$. Введем скалярную функцию $(U(t, x), f(x))$ для $f \in C_{0}^{1}(\bar{\Omega})$. Ее прирашение представим в виде

$$
\left\langle\Delta_{h} U, f\right\rangle=(U(t+h, x)-U(t, x), f)=\int_{t}^{t+h}\left(U^{\prime}(\tau, x), f\right) d \tau=\left\langle U^{\prime}, v\right\rangle .
$$

Здесь функция $v$ задается следующими равенствами: $v=f$ для $\tau \in[t, t+h]$; $v=0$ для $\tau \notin[t, t+h]$. Очевидно, что $v \in X_{1}=L^{p}\left(0, T ; \stackrel{\circ}{W_{p}^{1}}(\Omega)\right)$, поэтому можно воспользоваться тождеством (а) леммы 3.7 , согласно которому $\left(\Delta_{h} U, f\right)=$ $-\sum_{i=1}^{n}\left\langle w_{i}, D_{i} v\right\rangle-\left\langle w_{0}, v\right\rangle$. Отсюда

$$
\left|\left(\Delta_{h} U, f\right)\right| \leqslant \sum_{i=1}^{n}\left\|w_{i}\right\|_{L^{p^{\prime}}(Q)}\left\|D_{i} v\right\|_{L^{p}(Q)}+\left\|w_{0}\right\|_{L^{q^{\prime}}(Q)}\|v\|_{L^{q}(Q)} .
$$

Учитывая определение функции $v$, находим, что

$$
\begin{aligned}
\left\|D_{i} v\right\|_{L^{p}(Q)}^{p} & =\int_{t}^{t+h}\left\|D_{i} f\right\|_{L^{p}(\Omega)}^{p} d \tau=h\left\|D_{i} f\right\|_{L^{p}(\Omega)}^{p} \\
\|v\|_{L^{q}(Q)}^{q} & =\int_{t}^{t+h}\|f\|_{L^{q}(\Omega)}^{q} d \tau=h\|f\|_{L^{q}(\Omega)}^{q} .
\end{aligned}
$$

Отсюда следует, что для $f \in C_{0}^{1}(\bar{\Omega})$

$$
\left|\left(\Delta_{h} U, f\right)\right| \leqslant C\left(h^{1 / p} \sum_{i=1}^{n}\left\|D_{i} f\right\|_{L^{p}(\Omega)}+h^{1 / q} \sum_{i=1}^{n}\|f\|_{L^{q}(\Omega)}\right),
$$

где постоянная $C$ не зависит от $h$. Тем самым установлена непрерывность функции $(U(t, x), f)$ при условии, что $f \in C_{0}^{1}(\bar{\Omega})$. Пусть теперь $f \in L^{r}(\Omega)$. Приблизим ее последовательностью $f_{k} \in C_{0}^{1}(\bar{\Omega})$, так что $\left\|f-f_{k}\right\|_{L^{r}(\Omega)} \rightarrow 0$ при $k \rightarrow \infty$. Рассматриваемое прирашение представим в виде

$$
\left(\Delta_{h} U, f\right)=\left(\Delta_{h} U, f-f_{k}\right)+\left(\Delta_{h} U, f_{k}\right) .
$$

Тогда

$$
\left|\left(\Delta_{h} U, f\right)\right| \leqslant\left(\|U(t+h, x)\|_{L^{r^{\prime}}(\Omega)}+\|U(t, x)\|_{L^{r^{\prime}}(\Omega)}\right)\left\|f-f_{k}\right\|_{L^{r}(\Omega)}+\left|\left(\Delta_{h} U, f_{k}\right)\right| .
$$

По лемме 3.6(c) $\|U(t, x)\|_{L^{r^{\prime}}(\Omega)} \leqslant C$, где постоянная не зависит от $t$. По заданному $\varepsilon>0$ подберем номер $k$ такой, что $2 C\left\|f-f_{k}\right\|_{L^{r}(\Omega)}<\varepsilon / 2$. Затем по выбранному $k$ подберем число $h$ столь малое, что $\left|\left(\Delta_{h} U, f_{k}\right)\right|<\varepsilon / 2$. Это возможно по формуле (3.15). В результате получим неравенство $\left|\left(\Delta_{h} U, f\right)\right|<\varepsilon$, которое доказывает непрерывность функции $(U(t, x), f)$ для произвольной $f \in L^{r}(\Omega)$. Этим проверено утверждение леммы о слабой непрерывности функции $U=B(u)$. Равенство $B(u(0, x))=B\left(u_{0}(x)\right)$ установлено в лемме $3.8(\mathrm{c})$, что завершает доказательство. 


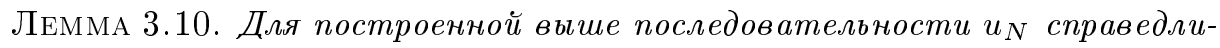
вы следующие предельные соотношения:

(a) $\lim _{N \rightarrow \infty} \int_{\Omega} \Gamma\left(u_{N}(0, x)\right) d x=\int_{\Omega} \Gamma(u(0, x)) d x ;$

(b) $\underline{\lim }_{N \rightarrow \infty} \int_{\Omega} \Gamma\left(u_{N}(T, x)\right) d x \geqslant \int_{\Omega} \Gamma(u(T, x)) d x$.

ДокАЗАТЕЛЬСтво. Вернемся к функции $\gamma(U)$, определенной в $\S 2$ равенством $\gamma(B(u))=\Gamma(u), u \in \mathbb{R}^{1}$. Построим по ней функционал $F(U)=\int_{\Omega} \gamma(U(x)) d x$. Согласно лемме 2.3 функция $\gamma(U)$ удовлетворяет неравенству $|\gamma(U)| \leqslant C\left(|U|^{r^{\prime}}+1\right)$, следовательно, введенный функционал непрерывен и локально ограничен на пространстве $L^{r^{\prime}}(\Omega)$. По лемме $3.8(\mathrm{~b})$ последовательность $B\left(u_{N}(0, x)\right) \rightarrow B(u(0, x))$ в пространстве $L^{r^{\prime}}(\Omega)$ при $N \rightarrow \infty$, поэтому в силу непрерывности функционала $F\left[B\left(u_{N}(0, x)\right)\right] \rightarrow F[B(u(0, x))]$, т.е.

$$
\lim _{N \rightarrow \infty} \int_{\Omega} \gamma\left[B\left(u_{N}(0, x)\right)\right] d x=\int_{\Omega} \gamma[B(u(0, x))] d x,
$$

что равнозначно пункту (а) леммы в силу равенства $\gamma(B(u))=\Gamma(u)$. Перейдем к (b). По лемме 2.3 функция $\gamma(U)$ является выпуклой и потому определяет выпуклый функционал $F(U)$. Каждый выпуклый локально ограниченный функционал слабо полунепрерывен снизу [18, с. 107]. Это означает следуюшее: если последовательность $V_{N} \rightarrow V$ слабо в пространстве $L^{r^{\prime}}(\Omega), \underline{\lim }_{N \rightarrow \infty} F\left(V_{N}\right) \geqslant F(V)$. Полагая здесь $V_{N}=B\left(u_{N}(T, x)\right), V=B(u(T, x))$ и используя лемму 3.8(a), получим

$$
\lim _{N \rightarrow \infty} \int_{\Omega} \gamma\left[B\left(u_{N}(T, x)\right)\right] d x \geqslant \int_{\Omega} \gamma[B(u(T, x))] d x
$$

что равносильно пункту (b) леммы, так как $\gamma(B(u))=\Gamma(u)$. Это завершает доказательство.

ЛЕмма 3.11. Для построенных выше последовательностей $u_{N}, U_{N}$ справедливо следующее соотношение

$$
\underset{N \rightarrow \infty}{\lim _{N \rightarrow \infty}}\left\langle U_{N}^{\prime}, u_{N}-v\right\rangle \geqslant\left\langle U^{\prime}, u-v\right\rangle ; \quad U=B(u), \quad v \in X
$$

ДокАЗАТЕЛЬСтво. Напомним, что $U_{N}^{\prime}=\left(\beta\left(u_{N}\right)+N^{-1}\right) u_{N}^{\prime}$. Отсюда прямым вычислением получаем формулу

$$
\begin{aligned}
\left\langle U_{N}^{\prime}, u_{N}\right\rangle= & \int_{\Omega} \Gamma\left(u_{N}(T, x)\right) d x-\int_{\Omega} \Gamma\left(u_{N}(0, x)\right) d x \\
& +\frac{1}{2 N}\left(\left\|u_{N}(T, x)\right\|_{L^{2}(\Omega)}^{2}-\left\|u_{N}(0, x)\right\|_{L^{2}(\Omega)}^{2}\right) .
\end{aligned}
$$

Так как $\left\|u_{N}(t, x)\right\|_{L^{2}(\Omega)} \leqslant c\left\|u_{N}(t, x)\right\|_{L^{r}(\Omega)} \leqslant C$ по лемме $3.1($ a), то в пределе при $N \rightarrow \infty$ согласно лемме 3.10 получим

$$
\varliminf_{N \rightarrow \infty}\left\langle U_{N}^{\prime}, u_{N}\right\rangle \geqslant \int_{\Omega} \Gamma(u(T, x)) d x-\int_{\Omega} \Gamma(u(0, x)) d x=\left\langle U^{\prime}, u\right\rangle
$$


Согласно лемме $3.7(\mathrm{~b}) \lim _{N \rightarrow \infty}\left\langle U_{N}^{\prime}, v\right\rangle=\left\langle U^{\prime}, v\right\rangle$ для каждого элемента $v \in Y_{0}$. Вычитая это соотношение из (3.16), получим

$$
\underset{N \rightarrow \infty}{\lim _{N}}\left\langle U_{N}^{\prime}, u_{N}-v\right\rangle \geqslant\left\langle U^{\prime}, u-v\right\rangle, \quad v \in Y_{0}
$$

Это есть требуемое неравенство для функций $v \in Y_{0}$. По непрерьвности оно продолжается на все функции $v \in X$, что завершает доказательство.

\section{§4. Предельный переход}

Приближенные уравнения (3.5) запишем в форме двойственности для функции $w \in Y_{N}:$

$$
\left\langle U_{N}^{\prime}, w\right\rangle+\sum_{i=1}^{n}\left\langle A_{i}\left(t, x, u_{N}, D u_{N}\right), D_{i} w\right\rangle+\left\langle A_{0}\left(t, x, u_{N}, D u_{N}\right), w\right\rangle=0 .
$$

Выберем $w=u_{N}-v$, где $v \in Y_{N}$, и представим уравнение в такой форме

$$
\begin{aligned}
& \left\langle U_{N}^{\prime}, u_{N}-v\right\rangle+\sum_{i=1}^{n}\left\langle A_{i}\left(t, x, u_{N}, D u_{N}\right)-A_{i}\left(t, x, u_{N}, D v\right), D_{i}\left(u_{N}-v\right)\right\rangle \\
& +\sum_{i=1}^{n}\left\langle A_{i}\left(t, x, u_{N}, D v\right), D_{i}\left(u_{N}-v\right)\right\rangle+\left\langle A_{0}\left(t, x, u_{N}, D u_{N}\right), u_{n}-v\right\rangle=0 .
\end{aligned}
$$

Перепишем это уравнение в условных обозначениях

$$
\left\langle U_{N}^{\prime}, u_{N}-v\right\rangle+\int_{Q} H\left(u_{N}, v\right) d Q+\int_{Q} G_{N} d Q+\int_{Q} F_{N} d Q=0 .
$$

Найдем предел каждого слагаемого при $N \rightarrow \infty$. Напомним, что предел первого слагаемого уже вычислен в лемме 3.11. Второе слагаемое неотрицательно в силу условия параболичности 2 А. Перейдем к последним слагаемым. При этом будет использовано следующее утверждение: если $f_{N} \rightarrow f$ в $X^{*}, w_{N} \rightarrow w$ слабо в $X$, то $\left\langle f_{N}, w_{N}\right\rangle \rightarrow\langle f, w\rangle$.

Лемма 4.1. Для последовательности $u_{N}$, построенной в предыдущем параграфе, справедливо следующее предельное соотночение

$$
\lim _{N \rightarrow \infty} \int_{Q} G_{N} d Q=\sum_{i=1}^{n}\left\langle A_{i}(t, x, u, D v), D_{i}(u-v)\right\rangle
$$


ДокАЗАТЕЛЬСТво. Перепишем рассматриваемый интеграл, используя обозначения леммы 2.8:

$$
\int_{Q} G_{N} d Q=\sum_{i=1}^{n}\left\langle z_{i N}, D_{i}\left(u_{N}-v\right)\right\rangle
$$

Так как $\left\|u_{N}\right\|_{X} \leqslant C$ согласно лемме $3.1(\mathrm{~b})$ и при этом $u_{N} \rightarrow u$ почти всюду в $Q$ по лемме $3.5(\mathrm{~b})$, то по лемме 2.8

$$
z_{i N}=A_{i}\left(t, x, u_{N}, D v\right) \rightarrow z_{i}=A_{i}(t, x, u, D v) \quad(N \rightarrow \infty)
$$

в норме пространства $L^{p^{\prime}}(Q)$. Одновременно последовательность $u_{N} \rightarrow u$ слабо в пространстве $X_{1}=L^{p}(0, T ; \stackrel{\circ}{W} \underset{p}{1}(\Omega))$ согласно лемме $3.6($ a). Это значит, что $D_{i} u_{N} \rightarrow D_{i} u$ слабо в пространстве $L^{p}(Q)$ при $N \rightarrow \infty$. Но тогда

$$
\left\langle z_{i N}, D_{i}\left(u_{N}-v\right)\right\rangle \rightarrow\left\langle z_{i}, D_{i}(u-v)\right\rangle \quad(N \rightarrow \infty)
$$

что составляет утверждение леммы.

Лемма 4.2. Введем обозначение

$$
\int_{Q} K_{N} d Q=\left\langle A_{0}\left(t, x, u_{N}, D u_{N}\right), u_{N}-u\right\rangle=\left\langle w_{0 N}, u_{N}-u\right\rangle
$$

Тогда

$$
\lim _{N \rightarrow \infty} \int_{Q} K_{N} d Q=0
$$

ДоКАЗАТЕЛЬСТво. Далее постоянно используется оценка $\left\|u_{N}\right\|_{X} \leqslant C$, полученная в лемме $3.1(\mathrm{~b})$. Пусть $E$ - измеримое подмножество области $Q, E^{\prime}$ - его дополнение. Разбив рассматриваемый интеграл на два по областям $E$ и $E^{\prime}$ соответственно, оценим его следующим образом

$$
\left|\int_{Q} K_{N} d Q\right| \leqslant\left\|w_{0 N}\right\|_{L^{q^{\prime}(E)}}\left\|u_{N}-u\right\|_{L^{q}(E)}+\left\|w_{0 N}\right\|_{L^{q^{\prime}\left(E^{\prime}\right)}}\left\|u_{N}-u\right\|_{L^{q}\left(E^{\prime}\right)}
$$

По заданному $\varepsilon>0$ найдем такое $\delta>0$, что $\int_{E}\left|w_{0 N}\right|^{q^{\prime}} d Q<\varepsilon^{q^{\prime}}$, как только mes $E<\delta$. Это возможно, так как функции под знаком интеграла имеют равностепенно абсолютно непрерывные интегралы по лемме 2.9. В результате можно оценить первую норму неравенства (4.4):

$$
\left\|w_{0 N}\right\|_{L^{q^{\prime}}(E)}=\left(\int_{E}\left|w_{0 N}\right|^{q^{\prime}} d Q\right)^{1 / q^{\prime}}<\varepsilon .
$$

Затем строим множество $E \subset Q$ с такими свойствами: $\operatorname{mes} E<\delta, u_{N} \rightarrow u$ равномерно на множестве $E^{\prime}$. Это возможно по теореме Егорова, так как $u_{N} \rightarrow u$ 
$(N \rightarrow \infty)$ почти всюду в $Q$ согласно лемме $3.5(\mathrm{~b})$. В частности, найдется номер $N_{0}$ такой, что

$$
\sup _{E^{\prime}}\left|u_{N}-u\right|<\varepsilon \quad\left(N>N_{0}\right) .
$$

В результате можно дать следующую оценку для номеров $N>N_{0}$ :

$$
\left\|u_{N}-u\right\|_{L^{q}\left(E^{\prime}\right)}=\left(\int_{E^{\prime}}\left|u_{N}-u\right|^{q} d Q\right)^{1 / q} \leqslant C \sup _{E^{\prime}}\left|u_{N}-u\right| \leqslant C \varepsilon .
$$

Далее,

$$
\left\|u_{N}-u\right\|_{L^{q}(E)} \leqslant\left\|u_{N}-u\right\|_{L^{q}(Q)} \leqslant c\left\|u_{N}-u\right\|_{X} \leqslant C
$$

согласно лемме 2.5 о непрерывности вложения $X$ в $L^{q}(Q)$ и лемме 3.1(b). Наконец,

$$
\left\|w_{0 N}\right\|_{L^{q^{\prime}}\left(E^{\prime}\right)} \leqslant\left\|w_{0 N}\right\|_{L^{q^{\prime}}(Q)} \leqslant C
$$

так как $w_{0 N} \rightarrow w_{0}$ слабо в $L^{q^{\prime}}(Q)$ по лемме 2.7. Подставляя полученные вьше соотношения в неравенстве (4.4), получим для $N>N_{0}:\left|\int_{Q} K_{N} d Q\right| \leqslant C \varepsilon$, где постоянная $C$ не зависит от $\varepsilon$. Так как $\varepsilon$ произвольно, то отсюда следует утверждение леммы.

ЛЕмма 4.3. Для последовательности $u_{N}$, построенной в третьем параграфе, справедливо следующее предельное соотношение

$$
\lim _{N \rightarrow \infty} \int_{Q} F_{N} d Q=\lim _{N \rightarrow \infty}\left\langle w_{0 N}, u_{N}-v\right\rangle=\left\langle w_{0}, u-v\right\rangle
$$

ДокАЗАТЕльство. Рассматриваемый интеграл представим в таком виде

$$
\int_{Q} F_{N} d Q=\left\langle w_{0 N}, u_{N}-u\right\rangle+\left\langle w_{0 N}, u-v\right\rangle
$$

Первое слагаемое справа стремится к нулю в силу леммы 4.2 , а второе имеет пределом число $\left\langle w_{0}, u-v\right\rangle$, так как $w_{0 N} \rightarrow w_{0}$ слабо в пространстве $L^{q^{\prime}}(Q)$ при $N \rightarrow \infty$ согласно лемме 2.7 , а $u-v \in L^{q}(Q)$ по лемме 2.5 о непрерывности вложения $X$ в $L^{q}(Q)$. В результате получается утверждение леммы.

Вернемся к равенству (4.2) в форме (4.3) и устремим $N$ к бесконечности. Учитьвая результаты лемм $3.11,4.1$ и 4.3 , получим в пределе

$$
\begin{aligned}
\left\langle U^{\prime}, u-v\right\rangle+\varlimsup_{N \rightarrow \infty} & \int_{Q} H\left(u_{N}, v\right) d Q \\
& +\sum_{i=1}^{n}\left\langle A_{i}(t, x, u, D v), D_{i}(u-v)\right\rangle+\left\langle w_{0}, u-v\right\rangle \leqslant 0 .
\end{aligned}
$$


Это неравенство получено при условии, что $v \in Y_{0}$. По непрерывности оно продолжается на все функции $v \in X$. В частности, можно взять $v=u$, что дает неравенство

$$
\varlimsup_{N \rightarrow \infty} \int_{Q} H\left(u_{N}, u\right) d Q \leqslant 0 .
$$

Однако по условию параболичности $2 \mathrm{~A} H\left(u_{N}, u\right) \geqslant 0$ почти всюду в $Q$. Отсюда следует, что $\lim _{N \rightarrow \infty} H\left(u_{N}, u\right)=0$ п.в. в $Q$, что в подробной записи имеет следующий вид: для почти всех $(t, x) \in Q$

$$
\lim _{N \rightarrow \infty} \sum_{i=1}^{n}\left[A_{i}\left(t, x, u_{N}, D u_{N}\right)-A_{i}\left(t, x, u_{N}, D u\right)\right]\left(D_{i} u_{N}-D_{i} u\right)=0 .
$$

Опираясь на это равенство, докажем следующее утверждение.

ЛЕмма 4.4. Для последовательности $u_{N}(t, x)$ справедливы следующие предельнье соотношения: $u_{N} \rightarrow u, D u_{N} \rightarrow D u$ почти всюду в $Q$ при $N \rightarrow \infty$.

ДокАЗАТЕЛЬСтво. Первое соотношение уже установлено ранеев лемме 3.5(b), поэтому проверим второе. Выберем точку $(t, x) \in Q$ так, чтобы выполнялись следующие условия: $u_{N}(t, x) \rightarrow u(t, x)$ при $N \rightarrow \infty ; D u(t, x)$ определено и конечно; функции $A_{i}(t, x, u, \xi)$ непрерывны по $u \in \mathbb{R}^{1}, \xi \in \mathbb{R}^{n}$ для $i=1, \ldots, n$. Каждое из этих свойств справедливо почти всюду в $Q$, поэтому их комбинация выполняется также для почти всех $(t, x) \in Q$. Для выбранной таким способом точки введем обозначения: $u_{N}=u_{N}(t, x), u=u(t, x), \xi_{N}=D u_{N}(t, x), \eta=D u(t, x)$. Введем также числовую последовательность

$$
H_{N}=\sum_{i=1}^{n}\left[A_{i}\left(t, x, u_{N}, \xi_{N}\right)-A_{i}\left(t, x, u_{N}, \eta\right)\right]\left(\xi_{i N}-\eta_{i}\right) .
$$

Исходя из формулы (4.6) и введенных обозначений, можно считать, что $H_{N} \rightarrow 0$ $(N \rightarrow \infty)$. Заметим также, что $H_{N}=H\left(t, x, u_{N}, \eta, \xi_{N}\right)$, где функция $H(t, x, u, \eta, \xi)$ введена в лемме 2.4. По условию $u_{N} \rightarrow u$ при $N \rightarrow \infty$, в частности, $\left|u_{N}\right| \leqslant M$ для некоторой постоянной $M$. Полагаем также $M_{1}=|\eta|$. Для указанных постоянных $M, M_{1}$ строим функцию $c(t, x)$ из леммы 2.4 , так что

$$
H_{N}=H\left(t, x, u_{N}, \eta, \xi_{N}\right) \geqslant c(t, x)\left|\xi_{N}-\eta\right|, \quad\left|\xi_{N}\right| \geqslant 2+M_{1} .
$$

Напомним, что функция $c(t, x)$ положительна почти всюду в $Q$. Считаем, что выбранная точка $(t, x) \in Q$ такова, что $c(t, x)>0$. Теперь имеется возможность выяснить свойства последовательности $\xi_{N}=D u_{N}(t, x)$. Допустим, что она неограничена, т.е. найдется подпоследовательность $\xi_{N k}$ такая, что $\left|\xi_{N k}\right| \rightarrow \infty$ при $k \rightarrow \infty$. Используя неравенство (4.7), получим

$$
H_{N_{k}} \geqslant c(t, x)\left|\xi_{N_{k}}-\eta\right| \rightarrow \infty \quad(k \rightarrow \infty) .
$$

Это противоречит условию, что $H_{N} \rightarrow 0$ при $N \rightarrow \infty$, следовательно, последовательность $\xi_{N}$ равномерно ограничена. Пусть $\xi$ - один из ее частичных пределов, 
т.е. $\xi_{N_{k}} \rightarrow \xi$ при $k \rightarrow \infty$. В силу непрерьвности функций $A_{i}(t, x, u, \xi)$ по двум последним аргументам получим

$$
\lim _{k \rightarrow \infty} H_{N_{k}}=\sum_{i=1}^{n}\left[A_{i}(t, x, u, \xi)-A_{i}(t, x, u, \eta)\right]\left(\xi_{i}-\eta_{i}\right)=0
$$

По условию параболичности $2 \mathrm{~A}$ такое равенство возможно только для $\xi=\eta$. Тем самьм любой частичный предел последовательности $\xi_{N}$ совпадает с $\eta$, что равносильно соотношению $D u_{N}(t, x) \rightarrow D u(t, x)$ при $N \rightarrow \infty$. Согласно построению это соотношение выполняется для почти всех $(t, x) \in Q$, что и утверждалось.

Объединяя доказанное утверждение с леммой 2.9, приходим к следующему заключению.

Лемма 4.5. В неравенстве (4.5) әлемент $w_{0}=A_{0}(t, x, u, D u)$.

ЛЕмма 4.6. Функиия $u(t, x)$ для любого $w \in X$ удовлетворяет уравнению

$$
\left\langle\frac{\partial}{\partial t} B(u), w\right\rangle+\sum_{i=1}^{n}\left\langle A_{i}(t, x, u, D u), D_{i} w\right\rangle+\left\langle A_{0}(t, x, u, D u), w\right\rangle=0 .
$$

ДокАЗАТЕльство. Вернемся к неравенству (4.5). Учитывая лемму 4.5 и условие параболичности $H\left(u_{N}, v\right) \geqslant 0$, получим для любого $v \in X$

$$
\left\langle U^{\prime}, u-v\right\rangle+\sum_{i=1}^{n}\left\langle A_{i}(t, x, u, D v), D_{i}(u-v)\right\rangle+\left\langle A_{0}(t, x, u, D u), u-v\right\rangle \leqslant 0
$$

Выберем $v=u-\lambda w$, где $\lambda>0, w \in X$, так что $u-v=\lambda w$. Подставив это выражение в (4.8) и разделив на $\lambda$, получим

$$
\left\langle U^{\prime}, w\right\rangle+\sum_{i=1}^{n}\left\langle A_{i}\left(t, x, u, D(u-\lambda w), D_{i} w\right)\right\rangle+\left\langle A_{0}(t, x, u, D u), w\right\rangle \leqslant 0 .
$$

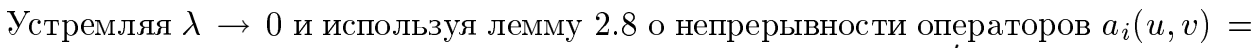
$A_{i}(t, x, u, D v)$ по второму аргументу как операторов из $X$ в $L^{p^{\prime}}(Q)$, придем к соотношению

$$
\left\langle U^{\prime}, w\right\rangle+\sum_{i=1}^{n}\left\langle A_{i}(t, x, u, D u), D_{i} w\right\rangle+\left\langle A_{0}(t, x, u, D u), w\right\rangle \leqslant 0
$$

Так как элемент $w \in X$ произволен, то вместо неравенства здесь можно ставить знак равенства. Полагая еще $U=B(u)$, придем к утверждению леммы.

Отметим, что получены все элементы теоремы 1.1, что дает возможность завершить ее доказательство. 
ДоКАЗАТЕЛЬСТво ТЕОРЕМЫ 1.1. Проверим, что выполнены все ее утверждения. Включение $u \in X$ установлено в лемме 3.6(а). Остальные утверждения пункта (а) теоремы вытекают из леммы 2.6 , в которой следует положить $u=v$. Пункт (b) теоремы совпадает с началом леммы 3.7. Пункт (c) составляет содержание леммы 4.6. Наконец, пункт (d) соответствует лемме 3.9. Это завершает доказательство теоремы.

В заключение приведем примеры функций $\beta(u)$, для которых применима развитая выше теория.

1. $\beta(u)=|u|^{\alpha}$, где $\alpha>0$. Показатель: $r=\alpha+2$.

2. $\beta(u)=1-u+u^{2}$. Очевидно, что

$$
\frac{1}{2} u^{2} \leqslant \beta(u) \leqslant 2\left(1+u^{2}\right)
$$

так что выполнено условие $1 \beta$ с показателем $r=4$.

3. Более общим образом можно взять $\beta(u)=\sum_{k=1}^{l} a_{k}|u|^{\alpha_{k}}$, где $\alpha_{k} \geqslant 0$, а числа $a_{k}$ таковы, что $\beta(u)>0$ при $u \neq 0$. Можно считать, что числа $\alpha_{k}$ расположены в порядке возрастания: $0 \leqslant \alpha_{1}<\alpha_{2}<\cdots<\alpha_{l}$. Нетрудно видеть, что тогда справедливы неравенства

$$
c|u|^{\alpha_{l}} \leqslant \beta(u) \leqslant C\left(|u|^{\alpha_{l}}+1\right) \quad(c>0),
$$

так что условие $1 \beta$ выполнено. Можно предложить и более сложные функции, например, дробно-рациональные.

Автор благодарит А.В. Иванова и С.И. Похожаева за полезное обсуждение результатов.

\section{Список литературы}

1. Лионс Ж.-Л. Некоторые методы решения нелинейных краевых задач. М.: Мир, 1972.

2. Raviart P. A. Sur la résolution de certaines équations paraboliques non linéaires // J. Funct. Anal. 1970. V. 5. № 2. P. 299-328.

3. Иванов А. В. Квазилинейные параболические уравнения, допускающие двойное вьрождение // Алгебра и анализ. 1992. Т. 4. №6. С. 114-130.

4. Калашников A.C. Некоторые вопросы качественной теории нелинейных вырождающихся параболических уравнений второго порядка // УМН. 1987. Т. 42. № 2. С. 135-176.

5. Alt H. W., Luckhaus S. Quasilinear elliptic-parabolic differential equations // Math. Z. 1983. V. 183. №3. P. 311-341.

6. Павлова М. Ф. Исследование уравнений нестационарной нелинейной фильтрации // Дифференц. уравнения. 1987. Т. 23. № 8. С. 1436-1446.

7. Леонов $K$. Я. О начально-краевой задаче для одного класса квазилинейных параболических уравнений. Препринт Института физики АН АзССР № 202. Баку: Институт физики АН АзССР, 1987.

8. Bernis $F$. Existence results for doubly nonlinear higher order parabolic equations on unbounded domains // Math. Ann. 1988. V. 279. P. 373-394.

9. Blanchard D., Francfort G.A. A few results on a class of degenerate parabolic equations // Ann. Sc. norm. super. Pisa. Cl. sci. 1991. V. 18. № 2. P. 213-219.

10. Blanchard D., Redwane $H$. Solutions renormalisées d'équations paraboliques à deux non linéarités // C. R. Acad. Sci. Ser. 1. 1994. V. 319. № 8. P. 831-835. 
11. Солтанов K. Н. Существование и несуществование глобального решения некоторых нелинейных эллиптико-параболических уравнений // Дифференц. уравнения. 1993. T. 29. № 4. C. 646-661.

12. Иванов А. В., Мкртыиян П. З. О существовании непрерывных по Гёльдеру обобщенных решений первой краевой задачи для квазилинейных параболических уравнений, допускающих двойное вырождение // Зап. научн. семин. ПОМИ. 1990. Т. 183. С. 5-28.

13. Иванов А. В., Мкртычян П. З., Яегер В. Существование и единственность регулярного решения первой начально-краевой задачи для некоторого класса дважды нелинейных параболических уравнений // Зап. научн. семин. ПОМИ. 1994. Т. 213. С. 48-65.

14. Гаевский X., Грёгер K., Захариас K. Нелинейные операторные уравнения и операторные дифференциальные уравнения. М.: Наука, 1978.

15. Ладызсенская О. А., Солонников В. А., Уральцева Н. Н. Линейные и квазилинейные уравнения параболического типа. М.: Наука, 1967.

16. Данфорд Н., Швари, Джс. Т. Линейные операторы. Общая теория. М.: ИЛ, 1962.

17. Никольский C. M. Приближение функций многих переменных и теоремы вложения. М.: Наука, 1977.

18. Вайнберг M. М. Вариационньй метод и методмонотонных операторов. М.: Наука, 1972.

Тульский государственньй университет

Поступила в редакцию

09.09.1996 